\title{
Probing Protein Motion Through Temperature Echoes
}

\author{
Klaus Schulten ${ }^{1,2}$, Hui Lu ${ }^{1,3}$, and Linsen Bai ${ }^{1,2}$ \\ 1 Beckman Institute, University of Illinois at Urbana-Champaign, Urbana, IL 61801, \\ USA \\ 2 Department of Physics, University of Illinois at Urbana-Champaign \\ 3 Department of Nuclear Engineering, University of Illinois at Urbana-Champaign
}

\begin{abstract}
In this tutorial lecture we demonstrate the existence of protein normal modes which maintain phase coherence for about one picosecond. We also study the dissipation of energy in proteins. The analysis is based on numerical experiments in which motional coherence, generated through two reassignments of Cartesian atomic velocities, induces echoes in the kinetic energy (temperature) and potential energy of proteins. Various echoes are produced in the case of the membrane protein bacteriorhodopsin. The echo phenomenon is then explained through a description of protein motion as an ensemble of harmonic oscillators. This description reveals that the echo can be expressed in terms of the temperature-temperature correlation function. A description in terms of Langevin oscillators allows one to account for decoherence effects. Finally, we consider echoes arising in an analytically tractable linear harmonic chain.
\end{abstract}

\section{Introduction}

Motions in proteins have been studied by observation and by molecular dynamics simulation for many years, but are still only poorly understood. In the case of solids and small molecules our understanding of characteristic motions is in a vastly better state, owing much to investigations of periodic motions in these systems $[1,2]$. Following this example researchers have studied normal modes in proteins. Normal mode analysis has been used, for example, to describe the fluctuations and to display concerted motions of proteins $[3,4,5,6,7,8,9]$. Normal modes have also been invoked to model slow motions between protein domains, for example, the hinge-bending motion of lysozyme [10, 11].

The widely adopted method to obtain normal modes for proteins is to calculate the second derivative (Hessian) matrix of the potential energy with respect to Cartesian coordinates or with respect to internal coordinates, and to diagonalize this matrix [3]. Due to anharmonic effects, normal modes defined through this method are not unique, but rather depend on the conformation of a protein. One would like to know how these modes, which are defined strictly only at $T=0$, manifest themselves at higher temperatures. The significant anharmonic contributions of force fields in proteins, such as torsional potentials, electrostatic and van der Waals interactions, call into question the existence of protein normal modes and even the existence of periodic or coherent motions.

In this lecture we apply a computational experiment to describe normal modes in proteins by temperature echoes. Such echoes were first observed in 


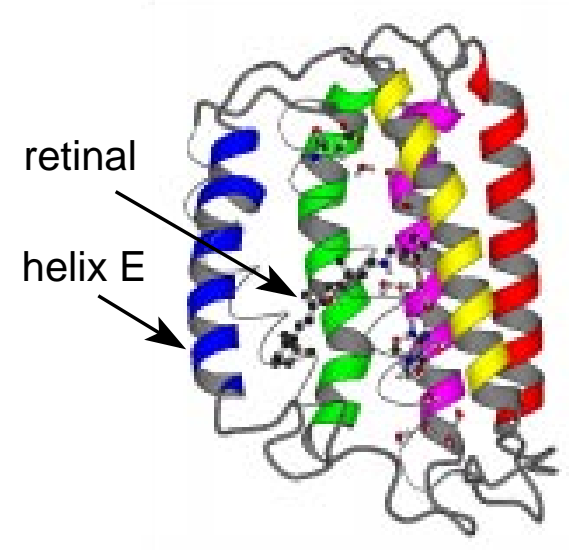

Fig. 1. Structure of the trans-membrane protein bacteriorhodopsin (from [12]). The protein has seven membrane-spanning helices A, B, C, .. G which form an elliptical cylinder around the chromophore retinal. Helices $\mathrm{C}$ and $\mathrm{D}$ are shown as thin lines to allow a view of the retinal. This protein serves to illustrate the various temperature echoes discussed in this lecture.

simulations of disordered solids by Grest et. al. [13, 14, 15, 16] and had been studied recently in the protein bovine pancreatic trypsin inhibitor (BPTI) in $[17,18]$. In these first studies echoes were generated through two quenches of the kinetic energy of the protein, i.e., through reassignment of zero velocities to all atoms of the protein at times $t=0$ and $t=\tau$. After the second quench one observes at time $2 \tau$ a dip in the temperature $T(t)$ which is defined through the total kinetic energy $E_{k}(t)$

$$
\begin{aligned}
T(t) & =\frac{2}{3 k_{B} N} E_{k}(t) \\
E_{k}(t) & =\sum_{i=1}^{N} \frac{1}{2} m_{i} \mathbf{v}_{i}^{2}(t) .
\end{aligned}
$$

Here $N$ denotes the number of atoms of the protein.

The lecture familiarizes the reader with several approaches to the study of protein motion. First, molecular dynamics simulations are utilized to induce and observe temperature echoes. Such simulations are by far the most frequent and most accurate tool for theoretical investigations of proteins. We will explain how molecular dynamics simulations produce echoes and will provide a few examples of echoes. Second, we model proteins as an ensemble of harmonic modes with a given frequency distribution and derive a representation which averages over initial thermal conditions. This normal mode analysis of temperature echoes is very idealistic, but has the benefit of a mostly analytical mathematical description which encapsulates the relationship between thermal fluctuations of 
protein motion and the echo phenomenon. We will recover the well-known result of linear response theory, which describes relaxation immediately after a perturbation through correlation functions of thermal fluctuations; however, we will express also echoes which, due to a build-up of motional coherence through two consecutive perturbations, occur long after the perturbations.

Normal mode analysis overestimates coherence and, hence, we introduce a representation of protein motion through an ensemble of Langevin oscillators, again for a given frequency distribution. This representation is still amenable to an analytical description which properly carries out averages over initial thermal conditions. We finally consider an extremely idealized model of protein modes in which the frequency distribution actually results from the dynamics, namely a chain of linearly coupled harmonic oscillators. Temperature echoes exemplify the combination of numerical simulation and statistical mechanical analysis which is invoked in the theory of protein dynamics. It may give the reader a view, however narrow, of the conceptual approaches utilized in the physics of proteins.

The lecture adopts the common style of theoretical physics textbooks in that all analytical results are derived in detail, starting from a basic knowledge in classical and statistical mechanics; in this respect the lecture should be particularly useful for students.

\section{Generating Echoes in the Protein Bacteriorhodopsin}

In order to produce coherent motion in a protein we follow the procedure suggested in [19]. The protein to be probed is bacteriorhodopsin, a seven helix trans-membrane protein shown in Fig. 1. The choice of the protein structure [12] was dictated by a desire to demonstrate that the echo phenomenon does not depend on the nature of the protein: in [19] a water soluble protein had been chosen, here we study a membrane protein with mainly $\alpha$-helical content. The protein was described through molecular dynamics (MD) simulations employing the program XPLOR [20] with the CHARMm force field [21]; all simulations employed the standard X-PLOR protein topology file topallh6x.pro and parameter file parmallh3x.pro to model bacteriorhodopsin. A dielectric constant of $\epsilon=1$ was assumed and an integration time step of 0.5 fs was selected.

The echo procedure is presented schematically in Fig. 2. One first equilibrates the protein in an MD simulation at a desired temperature $T_{0}$ by coupling to a heat bath through repeated rescaling of the velocities such that the temperature $T$ of the system, defined through Eqs. (1) and (2), assumes on average the value $T_{0}$. From then on all MD simulations are carried out in the microcanonical ensemble, i.e., energy is strictly conserved. This is a very important condition since motional coherence in a protein can be neither achieved nor maintained over any length of time if frictional and fluctuating forces are applied in any form.

Much of our attention in this lecture will be focussed on the temperature $T$ as defined in Eqs. (1) and (2) and, hence, we characterize the quantity a bit further. $T$ is actually fluctuating in time around its average value $T_{0}$ as demonstrated in 


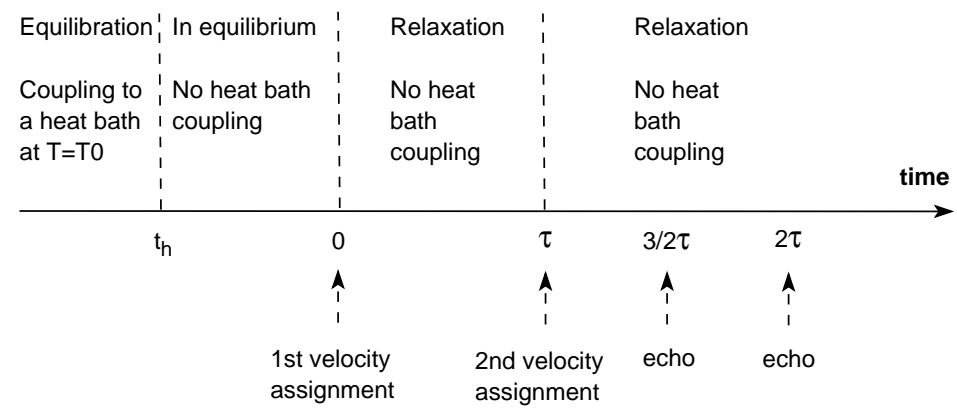

Fig. 2. Procedure of producing velocity reassignment echoes. No simulation is carried out until a coupling to a heat bath to achieve a desired temperature $T_{0}$ at $t=t_{h}<0$. The simulations are then continued in the microcanonical ensemble, beginning with equilibration, the reassignment of Cartesian velocities to all (or to a selected group of) protein atoms at $t=0$ using the set of velocities (4) followed by molecular dynamics simulation. At time $t=\tau$ atoms of the protein (all or a select group) are again reassigned Cartesian velocities, chosen from the set of velocities (7). Echoes arise, as described in the text, at times $\frac{3}{2} \tau$ and $2 \tau$.
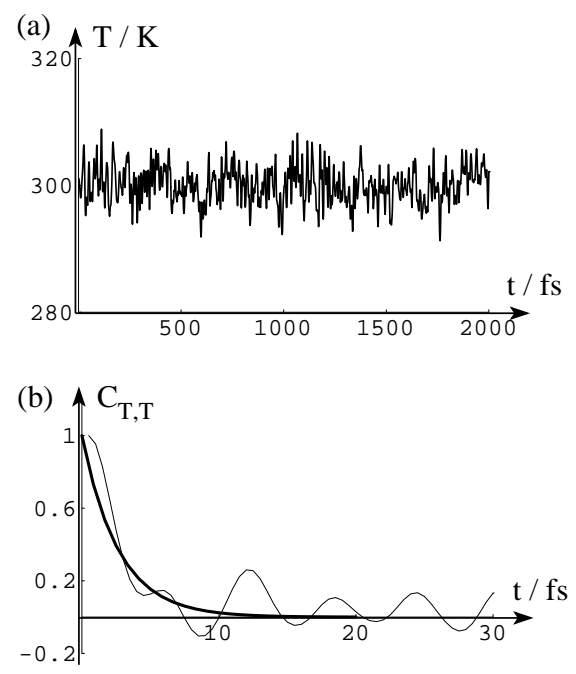

Fig. 3. (a) Temperature fluctuations in bacteriorhodopsin. The protein had been equilibrated at $T=300 \mathrm{~K}$ and $T(t)$ was evaluated according to Eqs. (1) and (2) and plotted. The fluctuations reflect dynamic properties of the protein. (b) Temperature-temperature correlation function $C_{T, T}(t)$ evaluated according to Eq. (3) from the trace of $T(t)$ as shown in (a) (thin solid line) and compared to a matched exponential $\exp \left(-t / \tau_{0}\right)$ for $\tau_{0}=2.67 \mathrm{fs}$ (thick solid line). 
Fig. 3. The fluctuations show a correlation in time which is conventionally characterized through the so-called temperature-temperature correlation function

$$
C_{T, T}(t)=\frac{\langle T(t) T(0)\rangle-\langle T(0)\rangle^{2}}{\left\langle[T(t)]^{2}\right\rangle-\langle T(0)\rangle^{2}}
$$

where $\langle\cdots\rangle$ denotes an ensemble average. One can recognize that $C_{T, T}$ decays to small values within about $5 \mathrm{fs}$, but then exhibits a slow decay of systematic oscillations. The behaviour of the protein hidden in these systematic oscillations is actually the main focus of this lecture. We seek to determine the dephasing time of these oscillations and want to inquire into the nature of the protein motions which remain coherent over periods actually much longer than shown in Fig. 3b.

In order to probe long time coherence in proteins one prepares an initial state in two steps as shown in Fig. 2. In a first step, at some instance defined through $t=0$, one prepares the system by assigning to the protein's $N$ atoms Cartesian velocities

$$
\mathcal{V}^{(1)}=\left\{v_{1}^{(1)}, v_{2}^{(1)}, v_{3}^{(1)}, \ldots, v_{3 N}^{(1)}\right\} .
$$

This can be realized readily by most MD simulation programs simply by restarting a simulation with the 'old' positions of a protein's atoms and with velocities assigned from the set (4). The velocities in (4) are chosen according to the Maxwell distribution at a temperature $T=T_{1}$, e.g., according to

$$
f\left(v_{i}^{(1)}\right)=\sqrt{\frac{m_{i}}{2 \pi k_{B} T_{1}}} \exp \left[-\frac{m_{i}\left(v_{i}^{(1)}\right)^{2}}{2 k_{B} T_{1}}\right] .
$$

$T_{1}$ can differ from the initial temperature $T_{0}$. For our first example we chose $T_{0}=300 \mathrm{~K}$ and $T_{1}=50 \mathrm{~K}$. After such assignment the protein has a potential energy content, determined by the 'old' atomic positions, of temperature $T_{0}$, and a kinetic energy content, determined by the 'new' velocities, of $T_{1}$. The subsequent dynamics will mix potential and kinetic energy such that the new temperature, measured again through Eqs. (1) and (2), will relax to a value of about $\frac{1}{2}\left(T_{0}+T_{1}\right)=175 \mathrm{~K}$. This relaxation is seen in Fig. 4a during the time period $0<t<100 \mathrm{fs}$, i.e., after the first velocity reassignment. Apparently, the protein exhibits random behaviour a few femtoseconds after the reassignment. One may ask what has been gained through the reassignment. The answer is: by assigning the velocities one knows at this point the phases of all protein modes; the phases are randomly distributed, but they are known through the set of velocities (4). This knowledge can be exploited in the second step of the procedure.

In fact, one stores the set (4) of velocities in memory and uses this information when, as before in the first step, a second set of random velocities

$$
\mathcal{V}^{(2)}=\left\{v_{1}^{(2)}, v_{2}^{(2)}, v_{3}^{(2)}, \ldots, v_{3 N}^{(2)}\right\}
$$



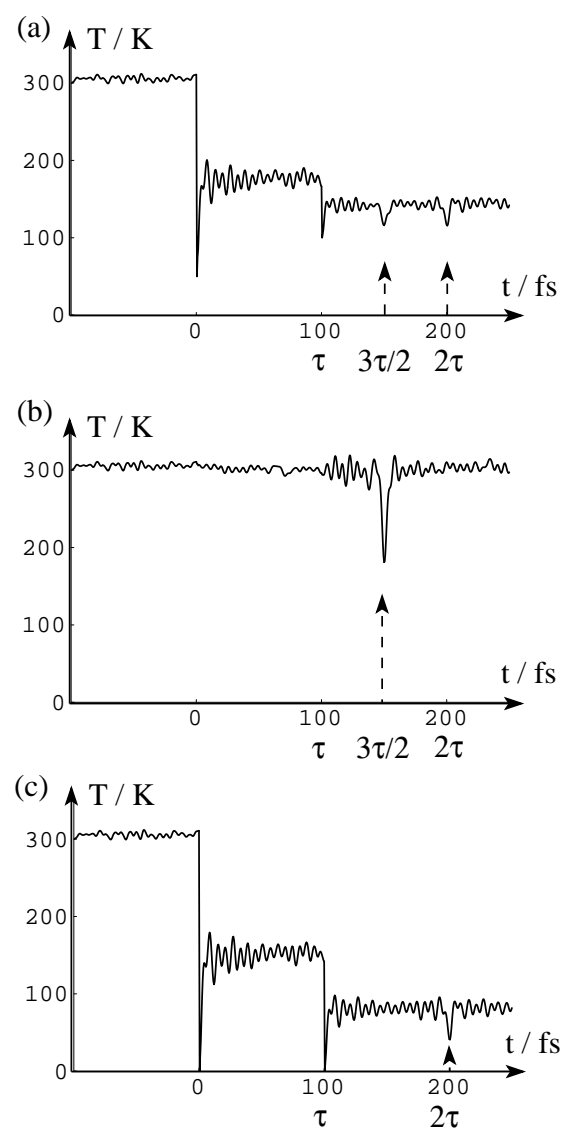

Fig. 4. Examples of temperature echoes in bacteriorhodopsin for $\tau=100$ fs. (a) Velocity reassignment echoes for $T_{0}=300 \mathrm{~K}, T_{1}=50 \mathrm{~K}, T_{2}=100 \mathrm{~K}$; the velocities assigned twice at $t=0$ and $t=\tau$ are strongly correlated according to (7). (b) Constant velocity reassignment echo, with $T_{0}=T_{1}=T_{2}=300 \mathrm{~K}$; identical velocities are assigned at $t=0$ and at $t=\tau$; a sole echo appears at $t=3 \tau / 2$. (c) Temperature quench echo for $T_{0}=300 K$; in this case the kinetic energy of the protein is quenched (all atomic velocities set to zero) at $t=0$ and at $t=\tau$; a sole echo appears at $t=2 \tau$.

is assigned to the protein's atoms at time $t=\tau$. This second set of velocities is selected strongly correlated to the velocities in (4), choosing

$$
\mathcal{V}^{(2)}=\left\{\lambda v_{1}^{(1)}, \lambda v_{2}^{(1)}, \lambda v_{3}^{(1)}, \ldots, \lambda v_{3 N}^{(1)}\right\}=\lambda \mathcal{V}^{(1)} .
$$

The new velocities obey again the Maxwell distribution (5), however, for a temperature $T_{2}=\lambda^{2} T_{1}$ as can be readily verified. For our first example we adopted $\lambda=\sqrt{2}$ corresponding to $T_{2}=100 \mathrm{~K}$ and $\tau=100 \mathrm{fs}$. The choice of velocities (7) induces indeed strong coherence in the protein's motion. This is born 


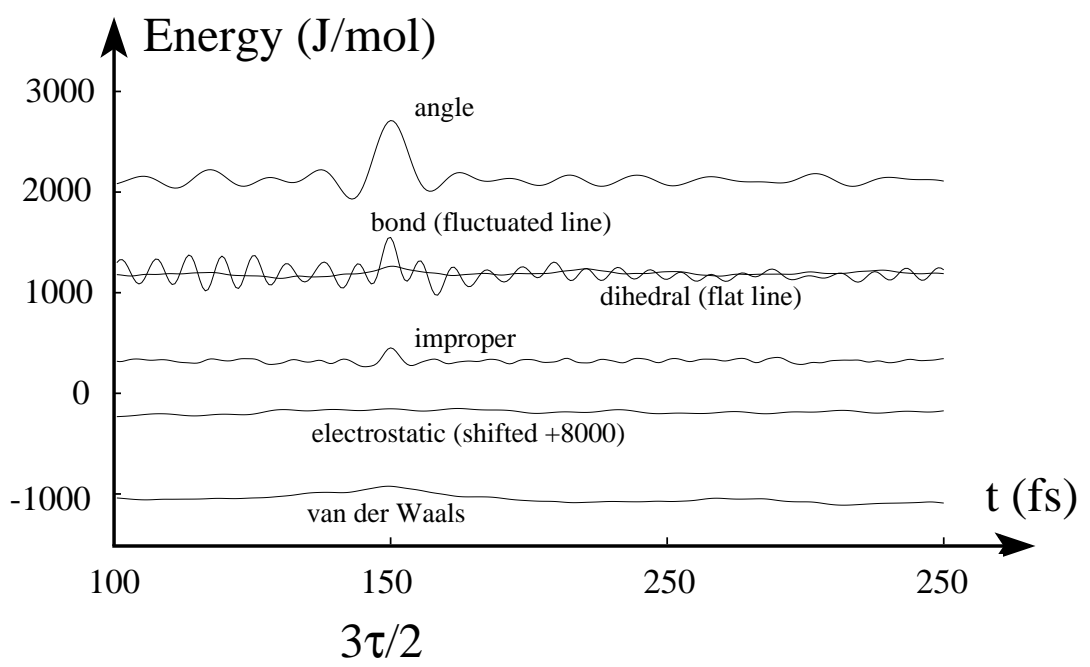

Fig. 5. Echoes reflected in the traces of the various potential energy contributions for bacteriorhodopsin prepared as in Fig. 4b. The potential energy contributions are defined in Eqs. (8-11). The dominant echo feature in the bond angle potential indicates that the normal modes involved in the echoes are skeletal motions.

out by the trace of the temperature $T(t)$ evaluated through Eqs. (1) and (2) and presented in Fig. 4a. After the reassignment of velocities the protein's kinetic energy corresponds to $T=100 \mathrm{~K}$ while the potential energy corresponds to $175 \mathrm{~K}$. Resurgence of the simulation leads to a rapid equilibration at a new temperature value of about $\frac{1}{2}\left(T_{1}+T_{2}\right)=137.5 \mathrm{~K}$. However, the systematic oscillations in $T(t)$ include now a hidden phase coherence which surfaces through two distinct features in the trace of $T(t)$, a dip at $t=3 \tau / 2$ and a dip at $t=2 \tau$, both of which can be clearly recognized in Fig. 4a. These dips will be referred to as temperature echoes.

Through the choice of the temperatures $T_{1}$ and $T_{2}$ and variation of the time $\tau$ one can alter the depths of the temperature echoes in Fig. 4a, but one cannot alter the instances at which the echoes arise. For example, choosing $T_{1}=T_{2}=$ $T_{0}$ the protein does not alter its temperature during the echo procedure. In this case an echo arises solely at $t=3 \tau / 2$ as shown in Fig. $4 \mathrm{~b}$; this echo is referred to as the constant temperature echo. Choosing $T_{1}=T_{2}=0$, a procedure called temperature quench, an echo arises solely at $t=2 \tau$; such an echo is demonstrated in Fig. 4c.

Conservation of energy implies $E_{k}(t)+V(t)=$ const where $V(t)$ is the total potential energy. As a result, one can monitor echoes not only through the temperature as defined in Eqs. (1) and (2), but also through the potential energy. On first sight this is unappealing since the total potential energy is more 
difficult to evaluate than the kinetic energy. However, this approach permits one to dissect the $V(t)$-echo into its various contributions, e.g., contributions from bond, van der Waals and electrostatic energies which allows one then to conclude which type of motions participate significantly in the normal modes underlying the echo effect. In fact, the potential energy of a protein, as employed in molecular dynamics simulations, is partitioned into so-called bond, angle, dihedral, improper, electrostatic and van der Waals contributions [21, 22]:

$$
V(t)=V_{\text {bond }}(t)+V_{\text {angle }}(t)+V_{\text {dihe }}(t)+V_{\text {impr }}(t)+V_{\text {elec }}(t)+V_{\text {vdw }}(t) .
$$

The bond energy and angle energy are described by quadratic functions

$$
V_{\text {bond }}=\sum_{\text {bonds }} k_{b}\left(|\mathbf{r}|-r_{0}\right)^{2} ; \quad V_{\text {angle }}=\sum_{\text {angles }} k_{a}\left(\theta-\theta_{0}\right)^{2} .
$$

The dihedral and improper terms can be expressed in a common functional form

$$
V_{\text {torsion }}= \begin{cases}\sum_{\text {torsions }} k_{\phi}\left[1+\cos \left(n \phi+\phi_{0}\right)\right] \quad(n=1,2,3, \ldots) \\ \sum_{\text {torsions }} k_{\phi}\left(\phi-\phi_{0}\right)^{2} .\end{cases}
$$

The electrostatic and van der Waals energies are given by the functions

$$
V_{\text {elec }}=\sum_{\text {pairs }} \frac{q_{1} q_{2}}{\epsilon r} ; \quad V_{\mathrm{vdw}}=\sum_{\text {pairs }}\left(\frac{A}{r^{12}}-\frac{B}{r^{6}}\right) .
$$

Figure 5 presents the traces of the various potential energy contributions in (8) as they arise after two constant temperature velocity reassignments for $\tau=100 \mathrm{fs}$. One can see that $V_{\text {angle }}(t)$ exhibits the largest contribution to the $3 \tau / 2-$ echo. The second largest contribution arises from $V_{\text {bond }}(t)$. The remaining potential energy terms contribute insignificantly, in particular, the electrostatic energy term shows no discernible contribution. One can interpret the results in Fig. 5 as a proof that the normal modes participating in the echoes for $\tau=100 \mathrm{fs}$ are skeletal motions involving bending vibrations (which affect bond angles) and, to a lesser degree, stretch vibrations.

One can also generate local echoes in proteins. To demonstrate this we have carried out a constant temperature velocity reassignment echo at $T=300 \mathrm{~K}$ and for $\tau=100$ fs by replacing twice only atomic velocities of subsegments of bacteriorhodopsin, leaving the velocities outside of those subsegments unaltered. Two examples are shown in Fig. 6. In one case we have produced an echo in the helix E of the protein (see Fig. 1) and in the second case in the chromophore retinal. The echo depth measures only about $80 \mathrm{~K}$ which is significantly less than that of an all-atom echo for the same $\tau$ (see Fig. 7).

The delay time $\tau$ between velocity reassignments determines the time scale over which $3 \tau / 2$ - and $2 \tau$-echoes arise. Naturally, dephasing of protein modes described by a time scale $\tau_{\text {dephase }}$, competes with the appearance of echoes since the latter rely on coherence of the motion. One expects that for $\tau>\tau_{\text {dephase }}$ echoes are weak. For this purpose, constant temperature all-atom echoes have been systematically generated for $\tau$-values increasing from a few femtoseconds 

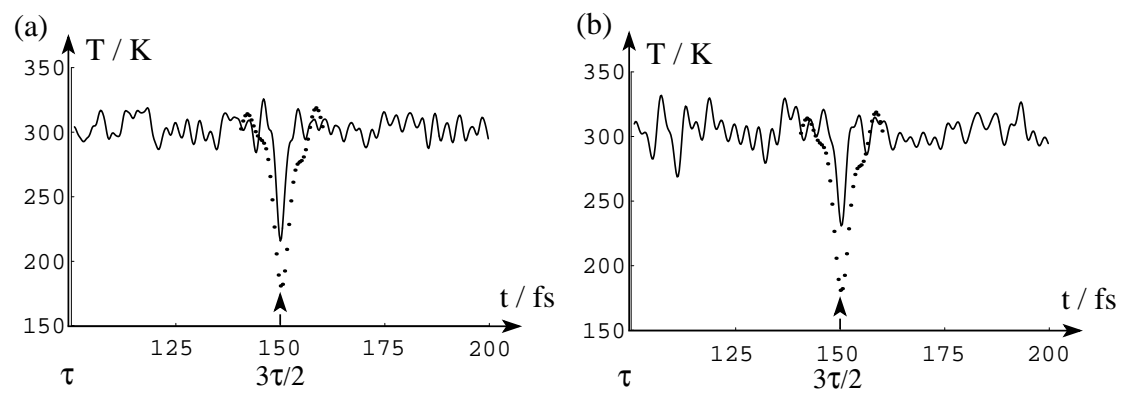

Fig. 6. Local velocity reassignment echoes (solid lines) in bacteriorhodopsin equilibrated at $T_{0}=300 \mathrm{~K}$ compared to all atom echoes (dotted lines). The protein has been prepared as for a constant temperature echo, i.e., with $T_{0}=T_{1}=T_{2}$. (a) Local echo involving all atoms of helix $\mathrm{E}$ of the protein (see Fig. 1); (b) Local echo involving all atoms of the chromophore retinal in the protein.

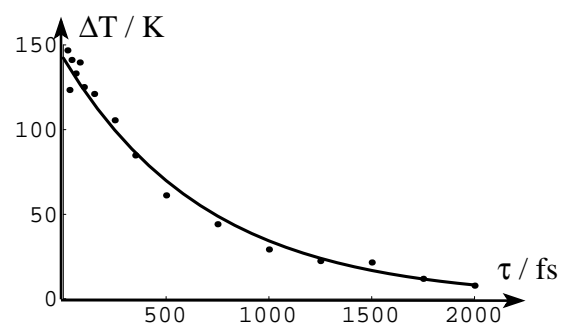

Fig. 7. Dependence of the depth of echo generated in bacteriorhodopsin for $T_{0}=T_{1}=T_{2}=300 \mathrm{~K}$ on the delay time $\tau$ between velocity reassignments. The simulation data are presented as dots and are matched to $\exp \left(-\tau / \tau_{\text {dephase }}\right)$ for $\tau_{\text {dephase }}=700 \mathrm{fs}$ (solid line).

to two picoseconds. The resulting depths of the echoes, presented in Fig. 7, show an exponential dependence on $\tau$ which apparently reflects an exponential decay of phase coherence. Remarkably, the results show that coherence of oscillations in proteins are maintained to a significant degree over a time of about a picosecond, a time which amounts to hundreds of periods of most bond stretch vibrations in proteins. Matching the $\tau$-dependence of the echo depth in Fig. 7 to an exponential $\exp \left(-\tau / \tau_{\text {dephase }}\right)$ yields $\tau_{\text {dephase }}=700 \mathrm{fs}$.

The temperature and potential energy echoes presented above are a signature of the motion of proteins on a 1 ps time scale. The value of the echoes hinges, however, on a suitable interpretation of the echo phenomena. This interpretation is furnished in the following two sections. 


\section{Harmonic Oscillator Description of Velocity Reassignment Echoes}

The echoes exhibited by the kinetic energy (temperature) and potential energy of a protein, after preparation of a coherent initial state, will be analyzed now in the framework of normal mode analysis. For this purpose the protein is considered an ensemble of uncoupled harmonic oscillators described by the Hamiltonians

$$
H_{\alpha}=\frac{p_{\alpha}^{2}}{2 m_{\alpha}}+\frac{1}{2} m_{\alpha} \omega_{\alpha}^{2} q_{\alpha}^{2}, \quad \alpha=1,2, \ldots
$$

From this we can solve the motion of the system

$$
\begin{gathered}
q_{\alpha}(t)=A_{\alpha} \cos \left(\omega_{\alpha} t+\theta_{\alpha}\right) \\
p_{\alpha}(t)=m_{\alpha} \frac{\mathrm{d} q_{\alpha}(t)}{\mathrm{d} t}=-m_{\alpha} A_{\alpha} \omega_{\alpha} \sin \left(\omega_{\alpha} t+\theta_{\alpha}\right) .
\end{gathered}
$$

This representation allows one to derive an analytical description of temperature echoes, as produced numerically in the previous section; in particular, one can deduce the relationship of echoes to other protein properties. However, normal mode analysis neglects decoherence effects.

\section{Simple Explanation of Temperature Echoes}

Before providing a systematic analysis we want to furnish a simple geometrical picture of the echo effect. For this purpose we notice that in a system with coordinates

$$
\begin{aligned}
\tilde{p}_{\alpha} & =p_{\alpha} / \sqrt{m_{\alpha}} \\
\tilde{q}_{\alpha} & =\sqrt{m_{\alpha}} \omega_{\alpha} q_{\alpha}
\end{aligned}
$$

the trajectory $\left(\tilde{q}_{\alpha}(t), \tilde{p}_{\alpha}(t)\right)$ of each oscillator $\alpha$ circumscribes a circle in a counter-clockwise direction with an angular frequency $\omega_{\alpha}$ and a radius $\sqrt{2 E_{\alpha}}$ where $E_{\alpha}$ is the energy of the oscillator; for an ensemble of oscillators in thermal equilibrium the energy assumes the average value $\left\langle E_{\alpha}\right\rangle=k_{B} T$. Figure 8a provides a snapshot of the oscillators in this presentation, indicating the circular trajectories for two oscillators. The reader should note that the individual oscillators precess with different angular velocities $\omega_{\alpha}$.

First velocity reassignment. We want to describe now the behaviour of the ensemble of oscillators. We consider the motion of the ensemble in the case of a temperature quench echo. Such an echo is prepared by setting in the initial preparation step, i.e., at $t=0$, all momenta $\tilde{p}_{\alpha}$ to zero. In the $\tilde{q}, \tilde{p}$-diagram the vectors characterizing the oscillators come to lie then all on the $\tilde{q}$-axis. Since there are many oscillators, we can divide them into subsets with the same en-

ergy. For the sake of simplicity we pick, as shown in Fig. 8b, that one subset of the oscillators, numbering 8 in this case, which have the same energy $E=E_{\alpha}$ : 
all circumscribe the same circle in the $\tilde{q}, \tilde{p}$-diagram, i.e., their trajectories are $\left(\tilde{q}_{\alpha}(t), \tilde{p}_{\alpha}(t)\right)=\left(\sqrt{2 E} \cos \omega_{\alpha} t, \sqrt{2 E} \sin \omega_{\alpha} t\right), \alpha=1,2, \ldots 8$. From now on we will follow only these 8 oscillators and show how the quench echo occurs for these oscillators. Other subsets can be treated using the same procedure as from Fig. 8b to Fig. 8f, but for different radii, i.e., different energies. It then follows for the same reason that the temperature of the ensemble, which is the total kinetic energy of all oscillators, will have an echo at time $2 \tau$.

Second velocity reassignment. The oscillators precess subsequently with different angular frequencies $\omega_{\alpha}, \alpha=1,2, \ldots 8$. A snapshot of the motion at time $t=\tau$ is shown in Fig. 8c; the isotropic spread of the vectors indicates that the motion is incoherent due to the random distribution of $\omega_{\alpha}$ values; the kinetic energy of the ensemble is $\frac{1}{2} \tilde{p}_{\alpha}^{2}=E \sin ^{2} \omega_{\alpha} \tau$, the average of which is $\frac{1}{2} E$, as expected. At this instance, i.e., at $t=\tau$, the ensemble of oscillators is quenched a second time by resetting $\tilde{p}_{\alpha}=0, \alpha=1,2, \ldots 8$ as shown in Fig. $8 \mathrm{~d}$; this aligns again, i.e., like at $t=0$, all vectors $\left(\tilde{q}_{\alpha}, \tilde{p}_{\alpha}\right), \alpha=1,2, \ldots 8$ with the $\tilde{q}_{-}$axis, however, now roughly half the vectors point to the right and half to the left; in fact, it holds $\left(\tilde{q}_{\alpha}(\tau), \tilde{p}_{\alpha}(\tau)\right)=\left(\sqrt{2 E} \cos \omega_{\alpha} \tau, 0\right)$. The energies of the ensemble are $E \cos ^{2} \omega_{\alpha} \tau$ and one expects the kinetic energy for the ensuing ensemble to be $\frac{1}{2} E\left\langle\cos ^{2} \omega_{\alpha} \tau\right\rangle_{\alpha}=\frac{1}{4} E$. The reduction of the average kinetic energy from $\frac{1}{2} E$ to $\frac{1}{4} E$ reflects the energy drained from the ensemble at $t=\tau$.

After the second velocity reassignment. At times $t>\tau$ the vectors $\left(\tilde{q}_{\alpha}, \tilde{p}_{\alpha}\right)$ spread uniformly again in both the $\tilde{q}-$ and $\tilde{p}$-directions as shown in Fig. 8e. The kinetic energies of the ensemble measure $E \cos ^{2} \omega_{\alpha} \tau \sin ^{2} \omega_{\alpha}(t-\tau), \alpha=1,2, \ldots 8$ or $\frac{1}{4} E\left[\sin \omega_{\alpha}(t-2 \tau)+\sin \omega_{\alpha} t\right]^{2}$. The average over all $\omega_{\alpha}$, indeed, yields a value $\frac{1}{4} E$, except near $t=2 \tau$ when the average reduces to $\frac{1}{8} E$. The latter feature corresponds to the echo shown in Fig. $4 \mathrm{~b}$. The $\left(\tilde{q}_{\alpha}, \tilde{p}_{\alpha}\right)$ vectors at time $t=2 \tau$ are shown in Fig. 8f. One can recognize that the vectors in Fig. $8 \mathrm{f}$ "concentrate" more closely around the $\tilde{q}$-axis than in case of Fig. 8 e, such that the kinetic energies are significantly smaller at $t=2 \tau$. In fact, the vectors at $t=2 \tau$ are given by $\left(\tilde{q}_{\alpha}(2 \tau), \tilde{p}_{\alpha}(2 \tau)\right)=\left(\sqrt{2 E} \cos ^{2} \omega_{\alpha} \tau, \sqrt{2 E} \cos \omega_{\alpha} \tau \sin \omega_{\alpha} \tau\right), \alpha=1,2, \ldots 8$ in which all $\tilde{q}-$ components are now positive as in the initial case (see Fig. $8 \mathrm{~b}$ ). This deviation from an isotropic symmetry originates from the orientation in Fig. $8 \mathrm{~b}$, such that a better sample in case of Fig. 8e would include the mirror image at the $\tilde{p}$-axis. However, even with that complement included, the kinetic energy of the ensemble is given by $\frac{1}{4} E \sin ^{2}\left(2 \omega_{\alpha} \tau\right), \alpha=1,2, \ldots 8$, the average of which measures only $\frac{1}{8} E$ at the $t=2 \tau$ instance. This implies an echo depth of $\frac{1}{4} E-\frac{1}{8} E=\frac{1}{8} E$. Another way to see the echo arising at the stage depicted in Fig. $8 \mathrm{f}$ is to notice that at $t=2 \tau$ every single mode goes back to the same phase or to same phase plus $\pi$ as in Fig. 8c. And in Fig. 8d, the modes near $\tilde{p}$-axis, such as modes 3 and 7 , lost more energy in the second quench than those modes near the $\tilde{q}$-axis, such as modes 1 and 6 . As a result the total kinetic energy, which is the sum of $\frac{1}{2} \tilde{p}_{\alpha}^{2}$, is lower in Fig. 8 f, i.e., at $t=2 \tau$, than in Fig. 8e, i.e., at any arbitrary $t>\tau$. 
We would like to note the following two points which emerge from the discussion of Fig. 8. First, the quench echo results from an average behavior of all the normal modes; every mode contributes to the echo feature. The echo depth, for an ensemble of harmonic oscillators, measures half of the current temperature. Second, the initial energies do not need to obey a Boltzmann distribution, rather, it is the distribution of oscillator frequencies that matters.

\section{Temperature Echo in the Harmonic Approximation}

We want to provide now a systematic derivation of velocity reassignment echoes which will provide us with an expression for the shape of the echoes as shown in Fig. 4. For this purpose we repeat the simple derivation above, carrying out, however, proper thermal averages and averages over the ensemble of frequencies $\omega_{\alpha}$. The present description assumes that the motion of a protein can be decomposed into a set of uncoupled normal modes described through the Hamiltonian in (12). The description follows closely the one given in [19]. For a protein with $\mathrm{N}$ atoms there exist $3 N-6$ different internal normal modes. Six degrees of freedom, which describe overall translation and rotation, are not counted as normal modes. We denote the frequency of the $\alpha$-th mode by $\omega_{\alpha}$, the corresponding effective mass by $m_{\alpha}$, and the associated vibrational coordinate by $q_{\alpha}$, where $\alpha=1,2, \ldots, 3 N-6$.

The position of the $\alpha$-th normal mode can be expressed as

$$
q_{\alpha}(t)=A_{\alpha} \cos \left(\omega_{\alpha} t+\theta_{\alpha}\right)
$$

with corresponding velocity

$$
u_{\alpha}(t)=\frac{\mathrm{d} q_{\alpha}(t)}{\mathrm{d} t}=-A_{\alpha} \omega_{\alpha} \sin \left(\omega_{\alpha} t+\theta_{\alpha}\right) .
$$

Here $\theta_{\alpha}$ are initial phases which are homogeneously distributed in the interval $[0,2 \pi] . A_{\alpha}$ are the initial amplitudes which are randomly distributed according to the Rayleigh distribution [23]

$$
P\left(A_{\alpha}\right)=\frac{m_{\alpha} \omega_{\alpha}^{2} A_{\alpha}}{k_{B} T_{0}} \exp \left(-\frac{m_{\alpha} \omega_{\alpha}^{2} A_{\alpha}^{2}}{2 k_{B} T_{0}}\right)
$$

where $T_{0}$ is the equilibrium temperature of the system. This distribution can be easily derived from the Boltzmann distribution of the energy. The total kinetic energy is

$$
E_{k}(t)=\sum_{\alpha} \frac{1}{2} m_{\alpha} \omega_{\alpha}^{2} A_{\alpha}^{2} \sin ^{2}\left(\omega_{\alpha} t+\theta_{\alpha}\right) .
$$

The kinetic energy averaged over the phases $\theta_{\alpha}$ is

$$
\left\langle E_{k}(t)\right\rangle_{\theta}=\left\langle\sum_{\alpha} \frac{1}{2} m_{\alpha} \omega_{\alpha}^{2} A_{\alpha}^{2} \sin ^{2}\left(\omega_{\alpha} t+\theta_{\alpha}\right)\right\rangle_{\theta}=\sum_{\alpha} \frac{1}{4} m_{\alpha} \omega_{\alpha}^{2} A_{\alpha}^{2} .
$$



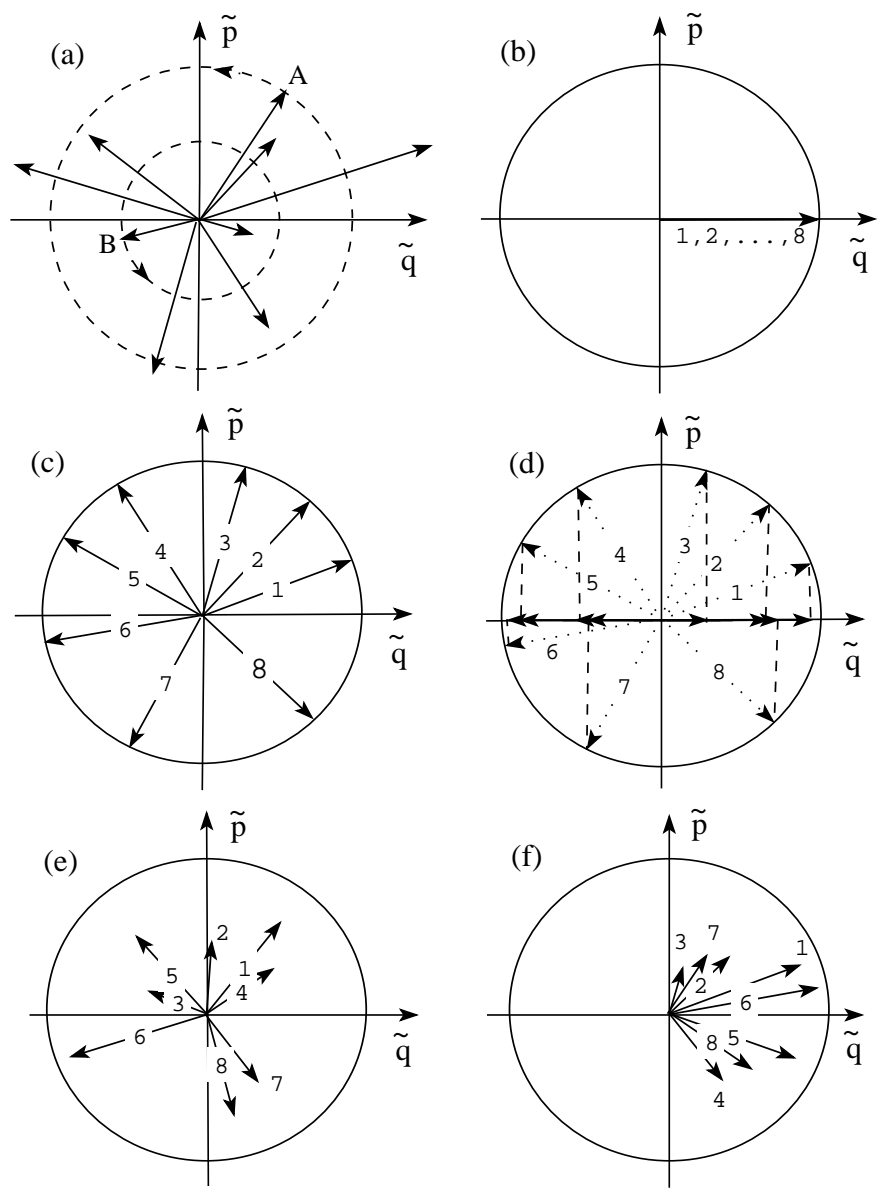

Fig. 8. Trajectories $\left(\tilde{q}_{\alpha}(t), \tilde{p}_{\alpha}(t)\right)$ of an ensemble of modes to demonstrate the temperature echo. The coordinates $\left(\tilde{q}_{\alpha}(t), \tilde{p}_{\alpha}(t)\right)$ are defined in Eqs. (15) and (16); (a) shows normal modes with different energies at $t<0$; for modes $\mathrm{A}$ and $\mathrm{B}$ the circles circumscribed by $\left(\tilde{q}_{\alpha}(t), \tilde{p}_{\alpha}(t)\right)$ are indicated by dashed lines; the modes precess around the origin with angular frequencies $\omega_{\alpha}$. (b) represents the state of eight normal modes at $t=0+$, after the initial quench of the kinetic energy at $t=0$; all momenta $\tilde{p}_{\alpha}(0)$ are set to zero; for the sake of simplicity we choose eight modes which now have the same initial energy, i.e., $\left(\tilde{q}_{\alpha}(t), \tilde{p}_{\alpha}(t)\right)$ begin to move on the same circle in a counterclockwise direction. (c) presents the normal modes at $t=\tau$-, the vectors $\left(\tilde{q}_{\alpha}(t), \tilde{p}_{\alpha}(t)\right)$ are distributed more or less homogeneously since the modes precess with different frequencies $\omega_{\alpha}$. At the instance $t=\tau$ the kinetic energy is quenched a second time, i.e., all momenta $\tilde{p}_{\alpha}$ are set to zero; the resulting states at $t=\tau+$ are shown in (d). (e) shows the modes at some arbitrary time $t>\tau$; the mode vectors $\left(\tilde{q}_{\alpha}(t), \tilde{p}_{\alpha}(t)\right)$ are again homogeneously distributed. However, at $t=2 \tau$ the vectors $\left(\tilde{q}_{\alpha}(2 \tau), \tilde{p}_{\alpha}(2 \tau)\right), \alpha=1,2, \ldots 8$ all point in the direction of the positive $\tilde{q}$ axis and are "focussed" near this axis, i.e., the momenta $\tilde{p}_{\alpha}(2 \tau)$ are small as shown in (f). 
Temperature-Temperature Correlation Function. Before calculating the echo we want to consider the thermal fluctuations of the temperature, defined through Eqs. (1) and (2) and discernable in the $T(t)$ traces in Figs. 4 and 6. An enlarged view of $\mathrm{T}(\mathrm{t})$ for bacteriorhodopsin at $T=300 \mathrm{~K}$ is presented in Fig. 3a. The time dependence of $T(t)$ can be characterized through the temperaturetemperature correlation function (3). We want to determine $C_{T, T}(t)$ for the ensemble of thermal oscillators described through Eqs. (17) and (18). For this purpose we express $C_{T, T}(t)$ through the kinetic energy using Eqs. (1) and (2)

$$
C_{T, T}(t)=\frac{\left\langle E_{k}(t) E_{k}(0)\right\rangle_{\theta}-\left\langle E_{k}(0)\right\rangle_{\theta}^{2}}{\left\langle\left[E_{k}(0)\right]^{2}\right\rangle_{\theta}-\left\langle E_{k}(0)\right\rangle_{\theta}^{2}}
$$

For the kinetic energy correlation function it holds that

$$
\begin{aligned}
& \left\langle E_{k}(t) E_{k}(0)\right\rangle_{\theta}= \\
& \left\langle\left(\sum_{\alpha} \frac{1}{2} m_{\alpha} \omega_{\alpha}^{2} A_{\alpha}^{2} \sin ^{2}\left(\omega_{\alpha} t+\theta_{\alpha}\right)\right)\left(\sum_{\lambda} \frac{1}{2} m_{\lambda} \omega_{\lambda}^{2} A_{\lambda}^{2} \sin ^{2} \theta_{\lambda}\right)\right\rangle_{\theta}
\end{aligned}
$$

where the summation over $\alpha$ and $\lambda$ is from 1 to $3 N-6$ and where $\langle\ldots\rangle_{\theta}$ denotes the average over the random phases $\theta_{\alpha}$ and $\theta_{\lambda}$. Replacing $\sin \theta$ by $\frac{1}{2 i}[\exp (i \theta)-$ $\exp (-i \theta)]$ and employing the averaging technique proposed by Rayleigh [23, 24],

$$
\left\langle\exp \left[ \pm i\left(\theta_{\alpha}+\theta_{\lambda}\right)\right]\right\rangle_{\theta}=0 ; \quad\left\langle\exp \left[ \pm i\left(\theta_{\alpha}-\theta_{\lambda}\right)\right]\right\rangle_{\theta}=\delta_{\alpha \lambda},
$$

one obtains

$$
\left\langle E_{k}(t) E_{k}(0)\right\rangle_{\theta}=\left(\sum_{\alpha} \frac{1}{4} m_{\alpha} \omega_{\alpha}^{2} A_{\alpha}^{2}\right)^{2}+\sum_{\alpha} \frac{1}{32} m_{\alpha}^{2} \omega_{\alpha}^{4} A_{\alpha}^{4} \cos \left(2 \omega_{\alpha} t\right) .
$$

Combining this result with (21) yields for (22)

$$
C_{T, T}(t)=\frac{\sum_{\alpha} m_{\alpha}^{2} \omega_{\alpha}^{4} A_{\alpha}^{4} \cos \left(2 \omega_{\alpha} t\right)}{\sum_{\alpha} m_{\alpha}^{2} \omega_{\alpha}^{4} A_{\alpha}^{4}} .
$$

For each molecular dynamics trajectory, the amplitudes $A_{\alpha}$ are constants of motion. However, as shown in [25], the correlation function $C_{T, T}(t)$ is almost identical for different trajectories. One may assume, therefore, that $C_{T, T}(t)$ calculated from one trajectory represents the temperature-temperature correlation function evaluated from the average over many trajectories with amplitudes $A_{\alpha}$ distributed according to Eq. (19). One may replace then in Eq. (26) the quantities $m_{\alpha}^{2} \omega_{\alpha}^{4} A_{\alpha}^{4}$ by their average values

$$
\left\langle m_{\alpha}^{2} \omega_{\alpha}^{4} A_{\alpha}^{4}\right\rangle_{A}=m_{\alpha}^{2} \omega_{\alpha}^{4} \int_{0}^{\infty} d A_{\alpha} A_{\alpha}^{4} p\left(A_{\alpha}\right)=8\left(k_{B} T_{0}\right)^{2} .
$$

Consequently, one obtains

$$
C_{T, T}(t)=\left\langle\cos \left(2 \omega_{\alpha} t\right)\right\rangle_{\alpha}
$$


where $\langle\ldots\rangle_{A}$ denotes the average over the amplitudes of the oscillators determined by use of Eq. (19), and where $\langle\ldots\rangle_{\alpha}$ is an average over all the normal modes, i.e.,

$$
\left\langle f\left(\omega_{\alpha}\right)\right\rangle_{\alpha}=\frac{1}{3 N-6} \sum_{\alpha} f\left(\omega_{\alpha}\right)=\int_{0}^{\infty} \mathrm{d} \omega D(\omega) f(\omega) .
$$

$D(\omega)$ denotes the normalized density of states.

The correlation function $C_{T, T}(t)$ could be evaluated from Eqs. (28) and (29) if the density of states $D(\omega)$ were known. Here we determine $C_{T, T}(t)$ according to Eq. (3) from molecular dynamics simulations, which do not necessarily satisfy the harmonic approximation. $C_{T, T}(t)$ was calculated from a $20 \mathrm{ps}$ interval in the simulation described in Section 2. The result is shown in Fig. 2. The correlation function can be matched to

$$
C_{T, T}(t) \approx e^{-t / \tau_{0}}, \tau_{0}=2.67 \mathrm{fs} .
$$

In the following, we will assume at various occasions

$$
C_{T, T}(t) \approx 0\left(t \gg \tau_{0}\right) .
$$

As is evident from Fig. 2, the exponential decay suggested in Eq. (30) is a rather poor approximation. The correlation function $C_{T, T}(t)$ has a long-time oscillatory behavior, which contains essential information on the density of states [18].

\section{Temperature-temperature correlation function and temperature quench response function ${ }^{1}$}

We want to demonstrate now that, in linear response theory, the response of a protein to a brief temperature pulse is related to the temperature-temperature correlation function. To demonstrate this relationship, we consider a single mode described by the Langevin equation

$$
m \ddot{x}=f(x)-\gamma \dot{x}+\sigma \xi .
$$

To this stochastic differential equation corresponds a Fokker-Planck equation $\partial_{t} p(x, v, t)=\mathcal{L}_{0}(x, v) p(x, v, t)$ where

$$
\mathcal{L}_{0}(x, v)=\frac{k_{B} T_{0} \gamma}{m^{2}} \partial_{v}^{2}+\frac{1}{m} \partial_{v}[\gamma v-f(x)]-\partial_{x} v .
$$

We denote by $p_{0}(x, v)$ the stationary position-velocity distribution for this mode for which holds $p_{0}(x, v) \sim \exp \left(-\frac{m v^{2}}{2 k_{B} T_{0}}\right)$, suppressing the $x$-dependence since it is immaterial in the following.

We consider now a perturbation applied to the mode at $t=0$ and described through the operator $\ell=\partial_{v}^{2}$, i.e., we consider the perturbation $\delta(t) \epsilon \partial_{v}^{2}$ for small

\footnotetext{
${ }^{1}$ As an introduction to the Langevin equations, Fokker-Planck equations and linear response theory employed here we recommend to the reader the relevant chapters in the monographs $[26,27]$.
} 
$\epsilon$. Adding this perturbation to the Fokker-Planck operator (33) corresponds to a sudden temperature pulse $\Delta T \delta(t)$ in the system, where

$$
\Delta T=\frac{m^{2} \epsilon}{k_{B} \gamma} .
$$

Application of $\ell$, for small $\epsilon$, induces a response in the kinetic energy $E_{k}$ described by $[28]$

$$
R_{E_{k}, \ell}(t)=\left\langle E_{k}(t) A(0)\right\rangle,
$$

the so-called response function. Here $A$ is

$$
A=p_{0}^{-1}(x, v) \ell p_{0}(x, v) .
$$

and, in the present case, is given by

$$
A=-\frac{m}{k_{B} T_{0}}+\frac{2 m}{\left(k_{B} T_{0}\right)^{2}}\left(\frac{1}{2} m v^{2}\right) .
$$

Hence, one can express the response to a brief temperature pulse through

$$
\begin{aligned}
R_{E_{k}, \ell}(t) & =-\frac{m}{k_{B} T_{0}}\left\langle E_{k}(t)\right\rangle+\frac{2 m}{\left(k_{B} T_{0}\right)^{2}}\left\langle E_{k}(t) E_{k}(0)\right\rangle \\
& =-\frac{m}{2}+\frac{2 m}{\left(k_{B} T_{0}\right)^{2}}\left\langle E_{k}(t) E_{k}(0)\right\rangle .
\end{aligned}
$$

Following the derivation of Eq. (28) one can state

$$
C_{T, T}(t)=\frac{\left\langle E_{k}(t) E_{k}(0)\right\rangle}{8\left(k_{B} T_{0}\right)^{2}}-\frac{1}{32} .
$$

Comparing Eq. (38) and Eq. (39), one arrives at

$$
R_{E_{k}, \ell}(t)=16 m C_{T, T}(t) .
$$

Hence, we have demonstrated that the response of the protein mode to a temperature pulse $\Delta T \delta(t)$, described by $R_{E_{k}, l}(t)$, is equal to the temperaturetemperature correlation function $C_{T, T}(t)$. We expect, then, that the relaxation of the temperature of the system after velocity reassignments is described by the temperature-temperature correlation function (28). We will show now that the temperature echo can also be expressed in terms of $C_{T, T}(t)$ within the harmonic approximation. 
Three stages of normal mode dynamics. As shown in Figs. 4 and 6, echoes can be observed for a protein prepared through two velocity reassignments by monitoring $T(t)$. Obviously, one needs to evaluate the total kinetic energy of the protein. For the purpose of this evaluation, we consider in turn the three stages of the echo dynamics, (0) before the first velocity reassignment, (1) between the first and the second reassignment, and (2) after the second reassignment, as discussed in connection with Fig. 8.

Normal modes imply concerted motions in which many protein atoms participate. The modes are described through a linear transformation from atomic coordinates $X_{j}, j=12, \ldots, 3 N$ to normal mode coordinates $q_{\alpha}, \alpha=1,2, \ldots 3 N-6$

$$
q_{\alpha}=\sum_{j=1}^{3 N} S_{\alpha j}(t) X_{j} .
$$

We have indicated through a time-dependence of the transformation matrix $\mathbf{S}(t)$ that the normal modes in a non-harmonic system, like a protein, are not invariant in time. In fact, one expects that the modes in proteins vary in time and, consequently, that the matrix $\mathbf{S}(t)$ experiences significant changes while a protein moves across conformational substates [29]. Since the evolution of the transformation matrix $\mathbf{S}(t)$ is unknown it is, strictly speaking, impossible to carry the velocity correlation expressed in Eq. (7) over to a normal mode analysis. In fact, defining the normal mode velocities at $t=0$

$$
u_{\alpha}^{(1)}=\sum_{j=1}^{3 N} S_{\alpha j}(0) v_{j}^{(1)},
$$

and at $t=\tau$

$$
\tilde{u}_{\alpha}^{(2)}=\sum_{j=1}^{3 N} S_{\alpha j}(\tau) v_{j}^{(2)},
$$

the sets of velocities, which should be assigned to the normal modes, are

$$
\mathcal{U}^{(1)}=\left\{u_{1}^{(1)}, u_{2}^{(1)}, u_{3}^{(1)}, \ldots, u_{3 N-6}^{(1)}\right\}
$$

and

$$
\tilde{\mathcal{U}}^{(2)}=\left\{\tilde{u}_{1}^{(2)}, \tilde{u}_{2}^{(2)}, \tilde{u}_{3}^{(2)}, \ldots, \tilde{u}_{3 N-6}^{(2)}\right\}
$$

corresponding to the velocity sets (4) and (6), respectively. However, the lack of knowledge of $\mathbf{S}(\tau)$ forces us to rather employ, at time $t=\tau$, the velocities transformed by $\mathbf{S}(0)$

$$
u_{\alpha}^{(2)}=\sum_{j=1}^{3 N} S_{\alpha j}(0) v_{j}^{(2)}
$$

and, hence, the set

$$
\mathcal{U}^{(2)}=\left\{u_{1}^{(2)}, u_{2}^{(2)}, u_{3}^{(2)}, \ldots, u_{3 N-6}^{(2)}\right\}
$$


can be written, according to Eq. (7),

$$
\mathcal{U}^{(2)}=\left\{\lambda u_{1}^{(1)}, \lambda u_{2}^{(1)}, \lambda u_{3}^{(1)}, \ldots, \lambda u_{3 N-6}^{(1)}\right\}=\lambda \mathcal{U}^{(1)} .
$$

For the statistical characteristics of the velocities $u_{\alpha}^{(1)}$ and $u_{\alpha}^{(2)}$ and their correlation the transformation $\mathbf{S}(0)$ is immaterial. Since the reassigned velocities are characterized only through their average properties the transformation matrix $\mathbf{S}(0)$ is not required; one can apply the statistical characteristics directly to $u_{\alpha}^{(1)}$ and $u_{\alpha}^{(2)}$ without knowing the Cartesian velocities $v_{j}^{(1)}$ and $v_{j}^{(2)}$. However, the replacement $\mathbf{S}(\tau) \rightarrow \mathbf{S}(0)$ implies an error for the correlation of velocities, as described, for example, by Eq. (7). The correlation of two velocities $u_{\alpha}^{(1)}$ and $\tilde{u}_{\alpha}^{(2)}$ can be written, using (48),

$$
\left\langle u_{\alpha}^{(1)} \tilde{u}_{\alpha}^{(2)}\right\rangle_{u}=g_{\alpha}(\tau, T)\left\langle u_{\alpha}^{(1)} u_{\alpha}^{(2)}\right\rangle_{u}=g_{\alpha}(\tau, T) \lambda\left\langle\left[u_{\alpha}^{(1)}\right]^{2}\right\rangle_{u} .
$$

Here $g_{\alpha}(\tau, T)$ is a factor accounting for the difference between $\mathbf{S}(\tau)$ and $\mathbf{S}(0)$ and is a function of the time interval $\tau$ and temperature $T$. In the harmonic case, which is assumed in this section, $g_{\alpha}(\tau, T)=1$. But for proteins, due to anharmonic effects, one expects $0<g_{\alpha}(\tau, T)<1, g_{\alpha}(\tau, T)$ deviating more from unity for longer $\tau$.

(0) Before the first reassignment. During this stage, the position of the $\alpha$-th normal mode at $t<0$ can be expressed as

$$
q_{\alpha}^{(0)}(t)=A_{\alpha} \cos \left(\omega_{\alpha} t+\theta_{\alpha}\right)
$$

where $A_{\alpha}$ denotes the amplitude of the mode, distributed according to Eq. (19), and where $\theta_{\alpha}$ denotes the phase of the mode which is homogeneously distributed in $[0,2 \pi]$. Obviously, averaging over the phases yields

$$
\left\langle\cos \left(n \theta_{\alpha}\right)\right\rangle_{\theta}=0 ; n=1,2,3, \ldots,
$$

a result needed further below. The velocities corresponding to Eq. (50) are

$$
\frac{\mathrm{d} q_{\alpha}^{(0)}(t)}{\mathrm{d} t}=-A_{\alpha} \omega_{\alpha} \sin \left(\omega_{\alpha} t+\theta_{\alpha}\right)
$$

The temperature correlation function $C_{T, T}(t)$ during this stage is given by (28).

(1) After the first reassignment but before the second. During this stage, i. e., for $0 \leq t<\tau$, the position for the $\alpha$-th normal mode can be expressed as

$$
q_{\alpha}^{(1)}(t)=A_{\alpha}^{(1)} \cos \left(\omega_{\alpha} t+\theta_{\alpha}^{(1)}\right) .
$$

In this case, the amplitudes $A_{\alpha}^{(1)}$ and phases $\theta_{\alpha}^{(1)}$ are determined through two sets of conditions, namely, that the positions (53) at $t=0$ must match the corresponding expression (50), from which follows

$$
A_{\alpha}^{(1)} \cos \theta_{\alpha}^{(1)}=A_{\alpha} \cos \theta_{\alpha}
$$


and that the velocities for all modes $\alpha$ are assigned values from the set $\mathcal{U}^{(1)}$ [see Eq. (4)], which implies

$$
\frac{\mathrm{d} q_{\alpha}^{(1)}(0)}{\mathrm{d} t}=-A_{\alpha}^{(1)} \omega_{\alpha} \sin \theta_{\alpha}^{(1)}=u_{\alpha}^{(1)} .
$$

(2) After the second reassignment. During this stage, i.e., at $t \geq \tau$, the position of the $\alpha$-th normal mode can be expressed as

$$
q_{\alpha}^{(2)}(t)=A_{\alpha}^{(2)} \cos \left[\omega_{\alpha}(t-\tau)+\theta_{\alpha}^{(2)}\right],
$$

where amplitudes $A_{\alpha}^{(2)}$ and phases $\theta_{\alpha}^{(2)}$ follow from two conditions, a match of Eq. (56) with Eq. (53) at $t=\tau$, i. e., from

$$
A_{\alpha}^{(2)} \cos \theta_{\alpha}^{(2)}=A_{\alpha}^{(1)} \cos \left(\omega_{\alpha} \tau+\theta_{\alpha}^{(1)}\right),
$$

and from the reassignment of the velocities, i.e., from matching the velocities corresponding to $(56)$ to new velocities [c.f. $(45,48)]$

$$
\frac{\mathrm{d} q_{\alpha}^{(2)}(\tau)}{\mathrm{d} t}=-A_{\alpha}^{(2)} \omega_{\alpha} \sin \theta_{\alpha}^{(2)}=\lambda u_{\alpha}^{(1)} .
$$

Defining

$$
u_{\alpha}=u_{\alpha}^{(1)} / \lambda_{1},
$$

where $\lambda_{1}=\sqrt{T_{1} / T_{0}}$, one can restate Eq. (58)

$$
-A_{\alpha}^{(2)} \omega_{\alpha} \sin \theta_{\alpha}^{(2)}=\lambda_{2} u_{\alpha}
$$

where $\lambda_{2}=\lambda \lambda_{1}=\sqrt{T_{2} / T_{0}}$. We note that the velocities $u_{\alpha}$ satisfy

$$
\left\langle m_{\alpha} u_{\alpha}^{2}\right\rangle_{u}=k_{B} T_{0} .
$$

Equations (57) and (60) allow one to determine $A_{\alpha}^{(2)}$ and $\theta_{\alpha}^{(2)}$ and to describe the motion for $t \geq \tau$ according to (56).

\section{Expression for the Temperature Echoes}

One can now determine the kinetic energy after the velocity reassignments. The resulting kinetic energy is

$$
\begin{aligned}
E_{k}(t)= & \sum_{\alpha} \frac{1}{2} m_{\alpha} \omega_{\alpha}^{2}\left[A_{\alpha}^{(2)}\right]^{2} \sin ^{2}\left[\omega_{\alpha}(t-\tau)+\theta_{\alpha}^{(2)}\right] \\
= & \sum_{\alpha} \frac{1}{2} m_{\alpha} \omega_{\alpha}^{2}\left\{A_{\alpha}^{(2)} \sin \theta_{\alpha}^{(2)} \cos \left[\omega_{\alpha}(t-\tau)\right]\right. \\
& \left.+A_{\alpha}^{(2)} \cos \theta_{\alpha}^{(2)} \sin \left[\omega_{\alpha}(t-\tau)\right]\right\}^{2} .
\end{aligned}
$$


Using Eqs. (57) and (60), and then (54) and (55), one can express the amplitudes $A_{\alpha}^{(2)}$ and phases $\theta_{\alpha}^{(2)}$ in terms of the initial amplitudes $A_{\alpha}$ and phases $\theta_{\alpha}$ in Eq. (50) as well as through the velocities $u_{\alpha}$ introduced in Eq. (59). One obtains

$$
\begin{aligned}
& E_{k}(t)= \sum_{\alpha} \frac{1}{2} m_{\alpha} \omega_{\alpha}^{2}\left\{-\frac{\lambda_{2} u_{\alpha}}{\omega_{\alpha}} \cos \left[\omega_{\alpha}(t-\tau)\right]+\right. \\
&\left.A_{\alpha}^{(1)} \cos \left[\omega_{\alpha} \tau+\theta_{\alpha}^{(1)}\right] \sin \left[\omega_{\alpha}(t-\tau)\right]\right\}^{2} \\
&=\sum_{\alpha} \frac{1}{2} m_{\alpha} \omega_{\alpha}^{2}\left\{-\frac{\lambda_{2} u_{\alpha}}{\omega_{\alpha}} \cos \left[\omega_{\alpha}(t-\tau)\right]+\right. \\
&\left.\quad \sin \left[\omega_{\alpha}(t-\tau)\right]\left(\frac{\lambda_{1} u_{\alpha}}{\omega_{\alpha}} \sin \omega_{\alpha} \tau+A_{\alpha} \cos \theta_{\alpha} \cos \omega_{\alpha} \tau\right)\right\}^{2} .
\end{aligned}
$$

This expression needs to be averaged over $A_{\alpha}, \theta_{\alpha}$, and $u_{\alpha}$. Employing Eqs. (19), (51) and (61) one can carry out the necessary averages which results in

$$
\begin{aligned}
\left\langle E_{k}(t)\right\rangle= & {\left[\frac{(3 N-6) k_{B} T_{0}}{2}\right]\left\langle\frac{1+\lambda_{1}^{2}+2 \lambda_{2}^{2}}{4}+\frac{1-\lambda_{1}^{2}}{4} \cos \left(2 \omega_{\alpha} \tau\right)\right.} \\
& -\frac{1-\lambda_{1}^{2}}{8} \cos \left(2 \omega_{\alpha} t\right)-\frac{1+\lambda_{1}^{2}-2 \lambda_{2}^{2}}{4} \cos \left[2 \omega_{\alpha}(t-\tau)\right] \\
& -\frac{1-\lambda_{1}^{2}}{8} \cos \left[2 \omega_{\alpha}(t-2 \tau)\right]+\frac{\lambda_{1} \lambda_{2}}{2} \cos \left[2 \omega_{\alpha}\left(t-\frac{\tau}{2}\right)\right] \\
& \left.-\frac{\lambda_{1} \lambda_{2}}{2} \cos \left[2 \omega_{\alpha}\left(t-\frac{3 \tau}{2}\right)\right]\right\rangle_{\alpha}
\end{aligned}
$$

where $\langle\ldots\rangle_{\alpha}$ denotes the remaining average over all normal modes as described by Eq. (29). In the above derivation we have employed the property that the average of $u_{\alpha} A_{\alpha}$ over $A_{\alpha}$ and $u_{\alpha}$ vanishes.

At this point one can introduce the temperature-temperature correlation function expressed through Eq. (28). According to (28), one can replace all occurrences of $\left\langle\cos \left[2 \omega_{\alpha}\left(t-t^{\prime}\right)\right]\right\rangle_{\alpha}$ by $C_{T, T}\left(t-t^{\prime}\right)$. Using (65), one obtains then for $t \geq \tau$

$$
\begin{aligned}
T(t)= & T_{0}\left[\frac{1+\lambda_{1}^{2}+2 \lambda_{2}^{2}}{4}+\frac{1-\lambda_{1}^{2}}{4} C_{T, T}(\tau)-\frac{1-\lambda_{1}^{2}}{8} C_{T, T}(t)\right. \\
& -\frac{1+\lambda_{1}^{2}-2 \lambda_{2}^{2}}{4} C_{T, T}(t-\tau)-\frac{1-\lambda_{1}^{2}}{8} C_{T, T}(|t-2 \tau|) \\
& \left.+\frac{\lambda_{1} \lambda_{2}}{2} C_{T, T}\left(t-\frac{\tau}{2}\right)-\frac{\lambda_{1} \lambda_{2}}{2} C_{T, T}\left(\left|t-\frac{3 \tau}{2}\right|\right)\right]
\end{aligned}
$$

$C_{T, T}(t)$ decays on a time scale of $\tau_{0}$ [c.f. Eqs. (30) and (31) and Fig. 3] and, hence, we can note $C_{T, T}(\tau) \approx 0, C_{T, T}(t) \approx 0$ and $C_{T, T}\left(\left|t-\frac{\tau}{2}\right|\right) \approx 0$ when 


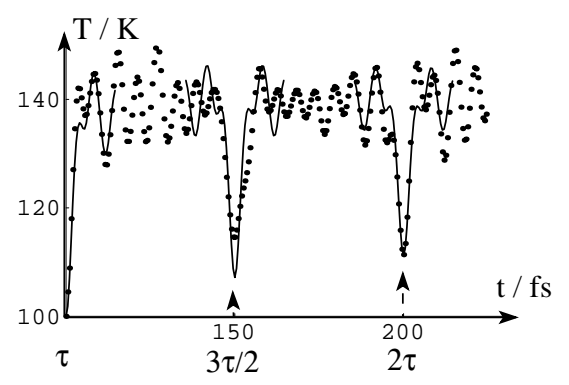

Fig. 9. Comparison of the kinetic energy of bacteriorhodopsin after velocity reassignments at $t=0$ and $t=\tau$ for $\tau=100 \mathrm{fs}$ and $T_{0}=300 \mathrm{~K}, T_{1}=50 \mathrm{~K}, T_{2}=100 \mathrm{~K}$ (same as Fig. 4) with the expression (67) (thin line); the simulated values $T(t)$ are shown as dots.

$t \geq \tau \gg \tau_{0}$. This leads to the expression

$$
\begin{aligned}
T(t) \approx T_{0} & {[\underbrace{\frac{1+\lambda_{1}^{2}+2 \lambda_{2}^{2}}{4}}_{\text {new temperature }}-\underbrace{\frac{1+\lambda_{1}^{2}-2 \lambda_{2}^{2}}{4} C_{T, T}(t-\tau)}_{\text {temperature recovery }}} \\
& -\underbrace{\frac{\lambda_{1} \lambda_{2}}{2} C_{T, T}\left(\left|t-\frac{3 \tau}{2}\right|\right)}_{\frac{3}{2} \tau \text {-pulse }}-\underbrace{\frac{1-\lambda_{1}^{2}}{8} C_{T, T}(|t-2 \tau|)}_{2 \tau \text {-pulse }}] .
\end{aligned}
$$

The terms in this expression can be interpreted in a straightforward way. We first note that the second, third, and fourth term do not contribute, except for $t \approx \tau, 3 \tau / 2,2 \tau$. The first term, accordingly, describes the average temperature after the second velocity reassignment. The second term describes the recovery of the temperature immediately after the second reassignment, i. e., at $t=\tau$. Since $C_{T, T}(0)=1$ the temperature at $t=\tau$, given by the first two terms in Eq. (67), is $T_{2}=\lambda_{2}^{2} T_{0}$, the expected result. The second term in Eq. (67) describes the relaxation of the temperature from this initial value to the average temperature $\frac{1}{4} T_{0}\left(1+\lambda_{1}^{2}+2 \lambda_{2}^{2}\right)$. This term conforms with Eq. (40) which states that the temperature recovery can be described by $C_{T, T}(t)$.

The third term in (67) describes the $\frac{3}{2} \tau$-pulse; the prefactor of $C_{T, T}(t)$ in this term is the depth $\widetilde{\Delta T}(3 \tau / 2)$ of this pulse, i. e.,

$$
\widetilde{\Delta T}\left(\frac{3 \tau}{2}\right)=\frac{T_{0}}{2} \lambda_{1} \lambda_{2}
$$

The fourth term describes the $2 \tau$-pulse; the depth of this pulse is

$$
\widetilde{\Delta T}(2 \tau)=\frac{T_{0}}{8}\left(1-\lambda_{1}^{2}\right) .
$$


Expression (67) not only correctly predicts the existence of echoes at $t=$ $3 \tau / 2$ and at $t=2 \tau$, but also provides a description of the detailed time dependence of the temperature recovery at $t=\tau$ and of the two echoes. Figure 9 demonstrates that Eq. (67) describes the temperature recovery and the echoes well. However, Eq. (67) predicts that the echo depths are independent of the delay time $\tau$ between velocity reassignments. This contradicts the results shown in Fig. 7, the deficiency stemming from the neglect of interactions between vibrational modes which give rise to dephasing. A theory which accounts for dephasing and correctly describes the $\tau$-dependence of the temperature echoes will be provided in the next section.

\section{Langevin Oscillator Description of Velocity Reassignment Echoes}

To account for dephasing as an explanation for a decrease of the echo depth with increasing $\tau$ we adopt in this section a description of protein motion in terms of an ensemble of Langevin oscillators. Such an ensemble is provided by the set of stochastic differential equations $[30,31]$

$$
\ddot{x}_{\alpha}+b_{\alpha} \dot{x}_{\alpha}+\omega_{\alpha}^{2} x_{\alpha}=\eta_{\alpha}(t), \quad \alpha=1,2, \ldots, 3 N-6 .
$$

In this model residual (anharmonic) interactions between normal modes are accounted for through dissipative (friction) and fluctuating forces $-b_{\alpha} \dot{x}_{\alpha}$ and $\eta_{\alpha}(t)$, respectively. These two terms are related through (fluctuation-dissipation theorem [26])

$$
\left\langle\eta_{\alpha}(0) \eta_{\alpha}(t)\right\rangle=2 k_{B} T_{0} b_{\alpha} \delta(t) / m_{\alpha},
$$

a property which ensures thermal equilibrium at temperature $T_{0}$. We assume that all modes are under-damped, i. e., $\omega_{\alpha}>\frac{1}{2} b_{\alpha}$, an assumption which we will justify below. Accordingly, we assume that the quantity

$$
\Omega_{\alpha}=\sqrt{\omega_{\alpha}^{2}-\frac{1}{4} b_{\alpha}^{2}}
$$

is real.

Time-dependent averages for a Langevin oscillator. To describe the temperature echo one needs to determine the average kinetic energy of the ensemble of Langevin oscillators (70) after two consecutive reassignments of velocities. For this purpose the average quantities $\langle x\rangle,\left\langle x^{2}\right\rangle,\langle v\rangle$ and $\left\langle v^{2}\right\rangle$ for a given initial position $x_{\alpha}(0)$ and velocity $v_{\alpha}(0)$ of Langevin oscillators will be required. We will demonstrate here the well known [32] evaluation of $\langle x\rangle$, leaving the evaluation of the remaining quantities as an exercise to the reader.

Ensemble averaging of Eq. (70) results in the equation

$$
\left\langle\ddot{x}_{\alpha}\right\rangle+b_{\alpha}\left\langle\dot{x}_{\alpha}\right\rangle+\omega_{\alpha}^{2}\left\langle x_{\alpha}\right\rangle=0 .
$$


We seek the corresponding equation for the Laplace transform

$$
\left\langle\hat{x}_{\alpha}\right\rangle=\int_{0}^{\infty} d t e^{-\omega t}\left\langle x_{\alpha}\right\rangle .
$$

In the case that the initial position of the oscillator is $x(0)=x_{o}$ and the initial velocity is $\dot{x}(0)=v_{o}$, it holds that

$$
\begin{aligned}
& \left\langle\hat{\dot{x}}_{\alpha}\right\rangle=\omega\left\langle\hat{x}_{\alpha}\right\rangle-x_{o} \\
& \left\langle\widehat{\ddot{x}}_{\alpha}\right\rangle=\omega^{2}\left\langle\hat{x}_{\alpha}\right\rangle-\omega x_{o}-v_{o} .
\end{aligned}
$$

Accordingly, Laplace transformation of Eq. (73) results in

$$
\omega^{2}\left\langle\hat{x}_{\alpha}\right\rangle-\omega x_{o}-v_{o}+b \omega\left\langle\hat{x}_{\alpha}\right\rangle-b x_{o}+\omega_{\alpha}^{2}\left\langle\hat{x}_{\alpha}\right\rangle=0
$$

which can be written, using Eq. (72),

$$
\left\langle\hat{x}_{\alpha}\right\rangle=\frac{\left(\omega+\frac{b}{2}\right) x_{o}}{\left(\omega+\frac{b}{2}\right)^{2}+\Omega^{2}}+\frac{\frac{b}{2} x_{o}+v_{o}}{\left(\omega+\frac{b}{2}\right)^{2}+\Omega^{2}} .
$$

Inverse Laplace transform yields for real $\Omega_{\alpha}$

$$
\left\langle x_{\alpha}(t)\right\rangle=x_{\alpha}(0) e^{-b_{\alpha} t / 2} A\left(\Omega_{\alpha} t\right)+\frac{v_{\alpha}(0)}{\Omega_{\alpha}} e^{-b_{\alpha} t / 2} \sin \left(\Omega_{\alpha} t\right)
$$

where we defined

$$
A\left(\Omega_{\alpha} t\right)=\cos \left(\Omega_{\alpha} t\right)+\frac{b_{\alpha}}{2 \Omega_{\alpha}} \sin \left(\Omega_{\alpha} t\right) .
$$

The remaining averages can be derived similarly and are given by (see Eq. (214) in [32])

$$
\begin{aligned}
\left\langle x_{\alpha}^{2}(t)\right\rangle & =\left\langle x_{\alpha}(t)\right\rangle^{2}+\frac{k_{B} T_{0}}{m_{\alpha} \omega_{\alpha}^{2}}\left(1-e^{-b_{\alpha} t} B\left(\Omega_{\alpha} t\right)\right) \\
\left\langle v_{\alpha}(t)\right\rangle & =-\frac{x_{\alpha}(0) \omega_{\alpha}^{2}}{\Omega_{\alpha}} e^{-b_{\alpha} t / 2} \sin \left(\Omega_{\alpha} t\right)+v_{\alpha}(0) e^{-b_{\alpha} t / 2} A\left(\Omega_{\alpha} t\right) \\
\left\langle v_{\alpha}^{2}(t)\right\rangle & =\left\langle v_{\alpha}(t)\right\rangle^{2}+\frac{k_{B} T_{0}}{m_{\alpha}}\left(1-e^{-b_{\alpha} t} B\left(\Omega_{\alpha} t\right)\right)
\end{aligned}
$$

where

$$
B\left(\Omega_{\alpha} t\right)=\frac{b_{\alpha}^{2}}{2 \Omega_{\alpha}^{2}} \sin ^{2}\left(\Omega_{\alpha} t\right)+\frac{b_{\alpha}}{2 \Omega_{\alpha}} \sin \left(2 \Omega_{\alpha} t\right)+1 .
$$

We will consider in the following the case of a constant temperature velocity reassignment echo for which one needs to assign twice the same velocities $u_{\alpha}$ to all modes. In order to determine the kinetic energy we consider again, in sequence, three periods: $(0)$ before the first velocity reassignment $(t<0),(1)$ after the first and before the second velocity reassignment $(0 \leq t<\tau)$, and (2) after the second velocity reassignment $(t \geq \tau)$. The derivation below follows again closely [19]. 
(0) Before the first velocity reassignment. This period does not need to be described in detail. All that required is information on the positions at the end of this period, namely, $x_{\alpha}^{(0)}(0)$. In fact, we solely need the average values

$$
\left\langle x_{\alpha}^{(0)}(0)\right\rangle_{(0)}=0,\left\langle\left[x_{\alpha}^{(0)}(0)\right]^{2}\right\rangle_{(0)}=\frac{k_{B} T_{0}}{m_{\alpha} \omega_{\alpha}^{2}}
$$

as becomes evident below. Here $\langle\ldots\rangle_{(0)}$ denotes the average for the system before the first reassignment. Since the velocities are reassigned at $t=0$, no information on the velocities $v_{\alpha}(0)$ is required.

(1) After the first reassignment but before the second. From this period of the dynamics again only the positions at time $t=\tau$, i. e., $x_{\alpha}^{(1)}(t)$, are required since the velocities will be reassigned. In fact, one needs solely the averages of $x_{\alpha}^{(1)}(t)$ and of $\left[x_{\alpha}^{(1)}(t)\right]^{2}$. Using Eqs. (79) and (81) one obtains for initial positions $x_{\alpha}^{(1)}(0)=x_{\alpha}^{(0)}(0)$ and the assigned velocities $v_{\alpha}^{(1)}(0)=u_{\alpha}$

$$
\begin{aligned}
\left\langle x_{\alpha}^{(1)}(\tau)\right\rangle_{(1)}= & x_{\alpha}^{(0)}(0) e^{-b_{\alpha} \tau / 2}\left[\cos \left(\Omega_{\alpha} \tau\right)+\frac{b_{\alpha}}{2 \Omega_{\alpha}} \sin \left(\Omega_{\alpha} \tau\right)\right] \\
& +\frac{u_{\alpha}}{\Omega_{\alpha}} e^{-b_{\alpha} \tau / 2} \sin \left(\Omega_{\alpha} \tau\right) \\
\left\langle\left[x_{\alpha}^{(1)}(\tau)\right]^{2}\right\rangle_{(1)}= & \left\langle x_{\alpha}^{(1)}(\tau)\right\rangle_{(1)}^{2}+\frac{k_{B} T_{0}}{m_{\alpha} \omega_{\alpha}^{2}}\{1- \\
& \left.e^{-b_{\alpha} \tau}\left[\frac{b_{\alpha}^{2}}{2 \Omega_{\alpha}^{2}} \sin ^{2}\left(\Omega_{\alpha} \tau\right)+\frac{b_{\alpha}}{2 \Omega_{\alpha}} \sin \left(2 \Omega_{\alpha} \tau\right)+1\right]\right\}
\end{aligned}
$$

In using these quantities below one needs to carry out the averages over all positions $x_{\alpha}^{(0)}(0)$, denoted by $\langle\cdots\rangle_{(0)}$, and over all velocities $u_{\alpha}$, denoted by $\langle\cdots\rangle_{u}$.

(2) After the second reassignment. From this period one seeks solely information on the velocities in order to determine the average kinetic energy. For specific initial positions $x_{\alpha}^{(2)}(\tau)=x_{\alpha}^{(1)}(\tau)$ and assigned velocities $v_{\alpha}^{(2)}(\tau)=u_{\alpha}$ it follows, using Eqs. (82) and (83), that

$$
\begin{aligned}
\left\langle v_{\alpha}^{(2)}(t)\right\rangle_{(2)}= & -\frac{x_{\alpha}^{(1)}(\tau) \omega_{\alpha}^{2}}{\Omega_{\alpha}} e^{-b_{\alpha}(t-\tau) / 2} \sin \Omega_{\alpha}(t-\tau) \\
& +u_{\alpha} e^{-b_{\alpha}(t-\tau) / 2}\left[\cos \Omega_{\alpha}(t-\tau)-\frac{b_{\alpha}}{2 \Omega_{\alpha}} \sin \Omega_{\alpha}(t-\tau)\right] \\
\left\langle\left[v_{\alpha}^{(2)}(t)\right]^{2}\right\rangle_{(2)}= & \left\langle v_{\alpha}^{(2)}(t)\right\rangle_{(2)}^{2}+\frac{k_{B} T_{0}}{m_{\alpha}}\left\{1-e^{-b_{\alpha}(t-\tau)}\left[\frac{b_{\alpha}^{2}}{2 \Omega_{\alpha}^{2}} \sin ^{2} \Omega_{\alpha}(t-\tau)\right.\right. \\
& \left.\left.-\frac{b_{\alpha}}{2 \Omega_{\alpha}} \sin 2 \Omega_{\alpha}(t-\tau)+1\right]\right\}
\end{aligned}
$$


In using these quantities below one needs to carry out the averages over all positions $x_{\alpha}^{(1)}(\tau)$, denoted by $\langle\cdots\rangle_{(1)}$, and over all velocities $u_{\alpha}$, denoted by $\langle\cdots\rangle_{u}$. Note that the velocities $u_{\alpha}$ must be averaged simultaneously for Eqs. (86-89) since the same velocities are assigned at $t=0$ and at $t=\tau$.

\section{Evaluation of the Temperature Echoes}

The average kinetic energy and, hence, the depth of the temperature echo, can be determined from Eqs. (88) and (89) after the following additional averages are taken:

1. average $\langle\cdots\rangle_{(1)}$ over all initial positions $x_{\alpha}^{(1)}(\tau)$, employing Eqs. (86) (87);

2. average $\langle\cdots\rangle_{(0)}$ over all initial positions $x_{\alpha}^{(1)}(0)$, employing Eq. (85);

3. average $\langle\cdots\rangle_{u}$ over all reassigned velocities $u_{\alpha}$ using $\left\langle u_{\alpha}\right\rangle=0$ and Eq. (61);

4. average $\langle\cdots\rangle_{\alpha}$ over all modes $\alpha$.

One obtains in this way for the echo depth

$$
\Delta T(3 \tau / 2)=T_{0}-\left\langle\left\langle\left\langle\left[m_{\alpha} v_{\alpha}^{(2)}(3 \tau / 2)\right]^{2} / k_{B}\right\rangle_{(2),(1),(0)}\right\rangle_{u}\right\rangle_{\alpha}
$$

or

$$
\begin{aligned}
\Delta T(3 \tau / 2)= & \frac{T_{0}}{2}\left\langlee ^ { - b _ { \alpha } \tau } \frac { \omega _ { \alpha } ^ { 2 } } { \Omega _ { \alpha } ^ { 2 } } \left[ 1-\cos \left(2 \Omega_{\alpha} \tau\right)-\frac{b_{\alpha}}{\Omega_{\alpha}} \sin \left(\Omega_{\alpha} \tau\right)+\right.\right. \\
& \left.\left.\frac{b_{\alpha}}{2 \Omega_{\alpha}} \sin \left(2 \Omega_{\alpha} \tau\right)\right]\right\rangle_{\alpha} .
\end{aligned}
$$

For $b_{\alpha}=0$ for all $\alpha$, i.e., for oscillators without friction and fluctuating forces, (91) yields a $\tau$-independent echo depth

$$
\Delta T(3 \tau / 2)=\frac{T_{0}}{2}\left[1-C_{T, T}(\tau)\right] \approx \frac{T_{0}}{2}
$$

which reproduces the result (68) derived for the harmonic model for $\lambda_{1}=$ $\lambda_{2}=1$.

In the case $b_{\alpha} \neq 0$, it holds that [33]

$$
\begin{aligned}
& \frac{\left\langle m_{\alpha} x_{\alpha}(0) x_{\alpha}(2 \tau)\right\rangle}{\left\langle m_{\alpha} x_{\alpha}^{2}\right\rangle}=e^{-b_{\alpha} \tau}\left[\cos \left(2 \Omega_{\alpha} \tau\right)+\frac{b_{\alpha}}{2 \Omega_{\alpha}} \sin \left(2 \Omega_{\alpha} \tau\right)\right], \\
& \frac{\left\langle m_{\alpha} v_{\alpha}(0) v_{\alpha}(2 \tau)\right\rangle}{\left\langle m_{\alpha} v_{\alpha}^{2}\right\rangle}=e^{-b_{\alpha} \tau}\left[\cos \left(2 \Omega_{\alpha} \tau\right)-\frac{b_{\alpha}}{2 \Omega_{\alpha}} \sin \left(2 \Omega_{\alpha} \tau\right)\right] .
\end{aligned}
$$

For $\tau \gg \tau_{0}$, both of the above quantities can be omitted, such that $\left\langle\frac{b_{\alpha}}{\Omega_{\alpha}} \sin \left(\Omega_{\alpha} \tau\right)\right\rangle_{\alpha}$ and $\left\langle\cos \left(2 \Omega_{\alpha} \tau\right)-\frac{b_{\alpha}}{2 \Omega_{\alpha}} \sin \left(2 \Omega_{\alpha} \tau\right)\right\rangle_{\alpha}$ can also be assumed to be negligible. Accordingly, we approximate $(91)$

$$
\Delta T(3 \tau / 2) \approx \frac{T_{0}}{2}\left\langle e^{-b_{\alpha} \tau} \frac{\omega_{\alpha}^{2}}{\Omega_{\alpha}^{2}}\right\rangle_{\alpha} .
$$


In the limit $b_{\alpha} \ll \omega_{\alpha}$ and choosing $b_{\alpha}$ the same constant $b_{0}$ for all $\alpha$, follows

$$
\Delta T(\tau) \approx \frac{T_{0}}{2} e^{-b_{0} \tau} .
$$

This expression for the echo depth predicts correctly the exponential dependence on $\tau$ which results from molecular dynamics simulations as shown in Fig. 7. Match of the simulation data yields a friction constant $b_{0}=1.43 \mathrm{ps}^{-1}$ at $T_{0}=300 \mathrm{~K}$. This value characterizes the vast majority of protein modes as underdamped, justifying our earlier assumption.

\section{Temperature Echo in a One-Dimensional Chain}

In our models above we have assumed ensembles of harmonic oscillators with given frequencies $\omega_{\alpha}$. These frequencies result, however, from the dynamics of harmonically coupled particles. The simplest case is a one-dimensional chain of $N$ atoms with momenta $p_{n}$ and positions $x_{n}$ governed by a Hamiltonian

$$
H=\sum_{n=1}^{N} \frac{p_{n}^{2}}{2 m}+\sum_{n=1}^{N+1} \frac{1}{2} m \tilde{\omega}^{2}\left(x_{n}-x_{n-1}\right)^{2}, \quad x_{0}, x_{N+1} \text { fixed } .
$$

The Hamiltonian can be expressed as a sum of quadratic forms $H=T+V+W$ where

$$
\begin{aligned}
T & =\sum_{n=1}^{N} t_{n n} p_{n}^{2}, \quad t_{n m}=\frac{1}{2 m} \delta_{n m} \\
V & =\sum_{n=1}^{N} v_{n n} x_{n}^{2}, \quad v_{n m}=m \tilde{\omega}^{2} \delta_{n m} \\
W & =\sum_{n, m=1}^{N} w_{n m} x_{n} x_{m}, \quad w_{n m}=-\frac{1}{2} m \tilde{\omega}^{2}\left(\delta_{n, m-1}+\delta_{n, m+1}\right) .
\end{aligned}
$$

Denoting by $\hat{t}, \hat{v}, \hat{w}$ the matrices with elements $t_{n m}, v_{n m}, w_{n m}$ one seeks a similarity transformation $\mathcal{A}$ which makes these matrices simultaneously diagonal. One can readily show that any $\mathcal{A}$ leaves $\tilde{t}=\mathcal{A} \hat{t} \mathcal{A}^{-1}$ and $\tilde{v}=\mathcal{A} \hat{v} \mathcal{A}^{-1}$ diagonal. Defining for $\alpha=1,2, \ldots N$

$$
\begin{aligned}
q_{\alpha} & =\sum_{n=1}^{N} \mathcal{A}_{\alpha n} x_{n} \\
\tilde{p}_{\alpha} & =\sum_{n=1}^{N} \mathcal{A}_{\alpha n} p_{n}
\end{aligned}
$$


one can, in fact, readily show

$$
\begin{aligned}
& T=\sum_{\alpha=1}^{N} \tilde{t}_{\alpha \alpha} \tilde{p}_{\alpha}^{2}, \quad \tilde{t}_{\alpha \beta}=\frac{1}{2 m} \delta_{\alpha \beta}, \\
& V=\sum_{\alpha=1}^{N} \tilde{v}_{\alpha \alpha} q_{\alpha}^{2}, \quad \tilde{v}_{\alpha \beta}=m \tilde{\omega}^{2} \delta_{\alpha \beta} .
\end{aligned}
$$

In order to render $\tilde{w}=\mathcal{A} \hat{w} \mathcal{A}^{-1}$ diagonal one chooses the well known matrix

$$
\mathcal{A}_{\alpha n}=\sqrt{\frac{2}{N}} \sin \frac{\alpha n \pi}{N+1} .
$$

One can verify

$$
W=\sum_{\alpha=1}^{N} \tilde{w}_{\alpha \alpha} q_{\alpha}^{2}, \quad \tilde{w}_{\alpha \beta}=m \tilde{\omega}^{2} \cos \frac{\alpha \pi}{N+1} \delta_{\alpha \beta} .
$$

This matrix is orthogonal, i.e., $\mathcal{A}^{T}$ is the inverse of $\mathcal{A}$. Using $1+\cos (\alpha \pi / N+1)=$ $2 \cos ^{2}(\alpha \pi / 2(N+1))$ one can finally write the Hamiltonian (96)

$$
H=\sum_{\alpha=1}^{N}\left(\frac{\tilde{p}_{\alpha}^{2}}{2 m}+\frac{1}{2} m \omega_{\alpha}^{2} q_{\alpha}^{2}\right)
$$

where

$$
\omega_{\alpha}=2 \tilde{\omega} \cos \frac{\alpha}{2(N+1)} .
$$

Having derived the normal modes we can now describe temperature echoes for the one-dimensional chain. We will consider in the following solely the case of temperature quench echoes. We adopt a description which applies both to all atom as well as to local echoes, i.e., we will quench the kinetic energy of atoms $n=1,2, \ldots r$ and observe the response of the particles $m=s \ldots t$ where $r \leq N$, $1 \leq s \leq N, s \leq t \leq N$.

(0) Before the first quench. Before the first quench, i.e., for $t<0$, the position and corresponding velocity of the $\alpha$-th normal mode can be expressed [c.f. Eqs. (50) and (52)]

$$
\begin{aligned}
& q_{\alpha}{ }^{(0)}(t)=A_{\alpha}{ }^{(0)} \cos \left(\omega_{\alpha} t+\theta_{\alpha}{ }^{(0)}\right) \\
& v_{\alpha}{ }^{(0)}(t)=-A_{\alpha}{ }^{(0)} \omega_{\alpha} \sin \left(\omega_{\alpha} t+\theta_{\alpha}{ }^{(0)}\right)
\end{aligned}
$$

where the amplitudes $A_{\alpha}{ }^{(0)}$ are distributed according to the Rayleigh distribution (19) and where the phases $\theta_{\alpha}{ }^{(0)}$ are homogenously distributed in the interval $[0,2 \pi]$. 

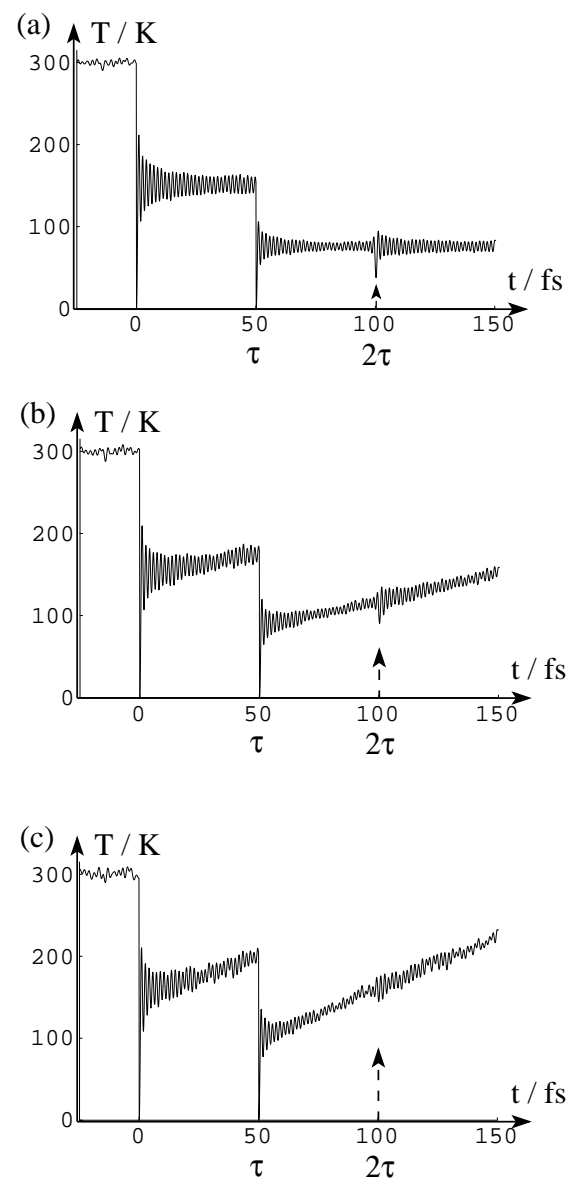

Fig. 10. Kinetic energy of a one-dimensional chain of 200 oscillators for total and partial quench at $t=0$ and $t=\tau$ with $t=100$ (arbitrary units) and $T_{0}=300 \mathrm{~K}$, $T_{1}=0, T_{2}=0$; (a) all oscillators quenched; (b) the first 100 oscillators quenched; shown is the kinetic energy of these oscillators; (c) the first 50 oscillators quenched; shown is the kinetic energy of these oscillators. In the calculations each mode had been sampled through 50 initial amplitudes and phases which were randomly assigned.

(1) After the first quench but before the second. One needs to express at this point the effect of the quench, executed on the velocities $\dot{x}_{n}$, in terms of the velocities $v_{\alpha}$ of the individual modes $\alpha$. For this purpose we define the quench matrix $\mathcal{Q}$

$$
\mathcal{Q}_{n m}=\left\{\begin{array}{l}
1 \text { if } n=m \text { and } r+1 \leq n \leq N \\
0 \text { otherwise }
\end{array}\right.
$$

which, applied to the vector of atomic velocities $\left(\dot{x}_{1}, \dot{x}_{2}, \ldots \dot{x}_{N}\right)^{T}$, describes the first quench. The effect of the quench on the normal mode velocities $v_{\alpha}$ is then 
described by

$$
\mathcal{B}=\mathcal{A} \mathcal{Q} \mathcal{A}^{T}
$$
form

We express the motion of the normal modes at $t>0$ in the by now familiar

$$
\begin{aligned}
& q_{\alpha}^{(1)}(t)=A_{\alpha}^{(1)} \cos \left(\omega_{\alpha} t+\theta_{\alpha}{ }^{(1)}\right) \\
& v_{\alpha}^{(1)}(t)=-A_{\alpha}{ }^{(1)} \omega_{\alpha} \sin \left(\omega_{\alpha} t+\theta_{\alpha}{ }^{(1)}\right) .
\end{aligned}
$$

The new amplitudes $A_{\alpha}{ }^{(1)}$ and phases $\theta_{\alpha}{ }^{(1)}$ are determined through the two conditions

$$
\begin{aligned}
& q_{\alpha}{ }^{(1)}(0)=q_{\alpha}{ }^{(0)}(0) \\
& v_{\alpha}{ }^{(1)}(0)=\sum \mathcal{B}_{\alpha \beta} v_{\beta}{ }^{(0)}(0) .
\end{aligned}
$$

From this follows

$$
\begin{aligned}
A_{\alpha}{ }^{(1)} \cos \left(\theta_{\alpha}{ }^{(1)}\right) & =A_{\alpha}{ }^{(0)} \cos \left(\theta_{\alpha}{ }^{(0)}\right) \\
-\omega_{\alpha} A_{\alpha}{ }^{(2)} \sin \left(\theta_{\alpha}{ }^{(1)}\right) & =-\sum_{\beta=1}^{N} \mathcal{B}_{\alpha \beta} \omega_{\beta} A_{\beta}{ }^{(0)} \sin \left(\theta_{\beta}{ }^{(0)}\right) .
\end{aligned}
$$

This yields for $A_{\alpha}{ }^{(1)}$ and $\theta_{\alpha}{ }^{(1)}$

$$
\begin{aligned}
& \theta_{\alpha}{ }^{(1)}=\arctan \left(\frac{\sum_{\beta=1}^{N} \mathcal{B}_{\alpha \beta} \omega_{\beta} A_{\beta}{ }^{(0)} \sin \left(\theta_{\beta}{ }^{(0)}\right)}{\omega_{\alpha} A_{\alpha}{ }^{(0)} \cos \left(\theta_{\alpha}{ }^{(0)}\right)}\right) \\
& A_{\alpha}{ }^{(1)}=\left\{\left[A_{\alpha}{ }^{(0)}\right] \cos ^{2}\left(\theta_{\alpha}{ }^{(0)}\right)+\left[\sum_{\beta=1}^{N} \mathcal{B}_{\alpha \beta} \omega_{\beta} A_{\beta}{ }^{(0)} \sin \left(\theta_{\beta}{ }^{(0)}\right) / \omega_{\alpha}\right]^{2}\right.
\end{aligned}
$$

(2) After the second quench. At $t=\tau$, the kinetic energy of the particles $1, \ldots, r$ is quenched again. Expressing

$$
\begin{aligned}
& q_{\alpha}{ }^{(2)}(t)=A_{\alpha}{ }^{(2)} \cos \left(\omega_{\alpha} t+\theta_{\alpha}{ }^{(2)}\right) \\
& v_{\alpha}{ }^{(2)}(t)=-A_{\alpha}{ }^{(2)} \omega_{\alpha} \sin \left(\omega_{\alpha} t+\theta_{\alpha}{ }^{(2)}\right)
\end{aligned}
$$

one can again determine the new amplitudes and phases from the conditions

$$
\begin{aligned}
& q_{\alpha}{ }^{(2)}(\tau)=q_{\alpha}{ }^{(1)}(\tau) \\
& v_{\alpha}{ }^{(2)}(\tau)=\sum \mathcal{B}_{\alpha \beta} v_{\beta}{ }^{(1)}(\tau)
\end{aligned}
$$


from which follows

$$
\begin{aligned}
& \theta_{\alpha}{ }^{(2)}=\arctan (\left.\frac{\sum_{\beta=1}^{N} \mathcal{B}_{\alpha \beta} \omega_{\beta} A_{\beta}^{(1)} \sin \left(\omega_{\beta} \tau+\theta_{\beta}{ }^{(1)}\right)}{\omega_{\alpha} A_{\alpha}{ }^{(1)} \cos \left(\omega_{\beta} \tau+\theta_{\alpha}{ }^{(1)}\right)}\right) \\
& A_{\alpha}{ }^{(2)}=\left\{\left[A_{\alpha}{ }^{(1)}\right] \cos ^{2}\left(\omega_{\alpha} \tau+\theta_{\alpha}{ }^{(1)}\right)+\right. \\
&\left.+\left[\sum_{\beta=1}^{N} \mathcal{B}_{\alpha \beta} \omega_{\beta} A_{\beta}{ }^{(1)} \sin \left(\omega_{\beta} \tau+\theta_{\beta}{ }^{(0)}\right) / \omega_{\alpha}\right]^{2}\right\}^{\frac{1}{2}} .
\end{aligned}
$$

The velocity and, hence, the kinetic energy of the chain at time $t>\tau$ can be determined from Eqs. (118), (119), (124) and (125). In the present case we focus on the temperature of the particles $n, s \leq n \leq t$ which is given by

$$
T(t)=\frac{2}{k_{B}(t-s+1)} \sum_{i=s}^{t} \frac{1}{2} m_{i} \dot{x}_{i}^{2}(t) .
$$

Echoes in one-dimensional chain. We have evaluated the all atom and local echoes for a linear harmonic chain of 200 atoms as described above. For this purpose we have carried out a numerical average, choosing initial amplitudes $A_{\alpha}^{(0)}$ and initial phases $\theta_{\alpha}^{(0)}$ employing suitable random number generators. We have employed samples of 50 such random choices. We employed Eqs. (118), (119), (124) and (125) to determine $A_{\alpha}^{(0)}$ and $\theta_{\alpha}^{(0)}$ and evaluated the temperature trace $T(t)$ using Eqs. (126) and (121). In this case atoms $1,2, \ldots M$ were quenched and monitored through their total kinetic energy. The resulting behaviour of $T(t)$ is shown in Fig. 10 for $M=200$ (all atom echo) as well as for $M=$ 100 and $M=50$. One can recognize that the local quenches produce only weak echoes in the present case, e.g., in case of $M=100,50$ the echo depth measures $37 \mathrm{~K}$ and $15 \mathrm{~K}$. This is due to the fact that in a homogenous linear chain vibrational modes extend over the complete chain. The appearance of stronger local echoes in proteins is due to the inhomogeneity of proteins which results in localized vibrational motion. One can recognize in Fig. 10 clearly the diffusion of temperature into the quenched part of the chain.

\section{A Word to Theoretical Physicists Contemplating Research in Biology}

The intellectual mastering of life at the molecular level naturally attracts physicists whose discipline has been so eminently successful in carrying the torch of mathematics into the natural sciences [34,35]. But physicists ought to be aware that there are differences as well as similarities in the quests towards physical and biological theory. The most important difference for a theoretical physicist to keep in mind in the pursuit of biological theory is that the latter is about advancing biology and not about advancing physics. A theoretical physicist must 
also realize that she or he is not as welcome by experimentalists in biology as she or he is in physics, since successes of theory in biology have been few, the field of evolutionary and hereditary biology being the notable exception. In fact, ever since the discovery of the structure of the double helix by Watson and Crick, which many molecular biologists considered a discovery snapped away from a deserving experimentalist who could have achieved it at her own pace, molecular biology has been skeptical about the role of theory. Recently, though, the standing of theory has dramatically improved, both due to challenges in the biological sciences which beg for theoretical approaches as well as due to the increasing and extremely supportive role of computation, e.g., for sequence analysis, structure determination and neural network modelling, in the biological "wet lab".

Challenges for theoretical work are many. Most pressing are needs for concepts and algorithms to handle and analyze the rapidly increasing genetic data bases. The complete genomes of several biological species, including homo sapiens, have recently become available or will soon become available. For many proteins, variants for numerous biological species are known with rich and still unearthed information underlying the conservation and variability of amino acids. The problem of predicting the structure of proteins from genetic sequences, the so-called protein folding problem, begs a solution with immeasurable opportunities once a solution is at hand. A further challenge is the structure-function relationship of proteins, a problem with extremely wide scope and characterized both through universality and diversity. An important class of proteins, so-called regulatory proteins, control the expression of the cell's genetic information through their capacity to recognize DNA sequences and alter local DNA packing. Of particular interest to physicists are biomolecular systems for which aggregates of biopolymers are the smallest functional units; examples of such systems are biological membranes and their complexes with proteins, motor proteins polymerizing and depolymerizing into strands controlling cell movement and intracellular transport, and the coats of viruses, spherical shells of hundreds of interlocking protein units which exist in a metastable state to disintegrate during the infection process.

The present lecture has focussed on proteins, actually only on a single one, bacteriorhodopsin. Theoretical studies of proteins began with the advent of sufficiently powerful computers to simulate large particle systems and with the explosive increase of atomic resolution structures of proteins. The latter structures, though necessary prerequisites, in themselves are not sufficient for any physical theory of protein function; the motions in a protein play an equal role. The sections above provide a narrow slice of the theory of protein motion. We felt that a detailed view of a narrow topic would serve better as an introduction to the field than a broad overview. However, the reader must bear in mind that the agenda of the field of protein dynamics is much broader. On the methodological side the field is concerned with providing accurate, yet simple force fields which govern the atomic motion of proteins. Ultimately, force fields will be determined in combined classical (for the nuclear motion)/quantum chemical (for the valence electrons) calculations employing, e.g., the Carr-Parinello method [36]. 
Researchers are investing currently strong efforts in developing efficient computational methods for classical dynamics of proteins involving tens to hundred thousands of atoms; a serious hurdle, for example, is the calculation of Coulomb forces since they need to be evaluated for all pairs of atoms for all time steps of the classical motion. Suitable integration schemes can economize the costly update of forces, in particular the Coulomb forces, with a resulting boost in computational efficiency [37]. At present a practitioner of the theory of proteins needs to be extremely competent in scientific computing with an understanding of massively parallel computing holding a particular promise.

On the conceptual side an entry to protein dynamics is provided by studying normal modes. Since the classical Hamiltonian describing atomic motion is significantly non-harmonic and also extremely heterogenous, conventional normal mode analysis as applied, e.g., for crystals, is not suitable. A quasi-harmonic description derives normal modes from a principal component analysis, i.e., from a diagonalization of the covariance matrix of all atomic positions, averaged over time. However, a gliding average with a, say, 100 ps window, reveals that modes derived in such way vary in time due to significant conformational transitions and disorder in proteins. Protein motion needs to be characterized also on spatial scales longer than atomic resolution, i.e., involving multi-atom segments of proteins. In fact, many proteins exhibit conformational changes which can be described as rotations of segments around hinges or as motions of flaps formed by secondary structure elements, e.g., $\alpha$-helices or loops between $\alpha$-helices.

The abstraction of functional dynamics from molecular dynamics simulations still remains an important challenge. Following established approaches one can try to identify correlation functions and susceptibilities which provide essential characteristics of protein dynamics and relate to observations and function: the dynamic structure function provides the Fourier transform of the motion of a protein's constituents and is observable through neutron diffraction or Mössbauer spectroscopy; the correlation function of the energy difference between two quantum states with diagonal coupling to the protein matrix accounts for the transition rate between the two states and, hence, for spectral or thermal transfer rates, e.g., the rate of electron transfer; the dielectric susceptibility and thermal susceptibility, determined through monitoring dipolar or energy fluctuations account for dielectric properties, e.g., at membrane surfaces, or specific heats, e.g., of ordered and disordered water.

The theory of proteins, as a relatively young field, can benefit tremendously from related and already established fields. The closest relative is the theory of liquids since solvent molecules, though not connected into a polymer and much more homogeneous in structure, are subject to similar forces and disorder phenomena (see [38]). On larger length and longer time scales, molecular hydrodynamics [39] can provide much guidance to gain understanding of low frequency motion of protein segments encompassing many atoms. Condensed matter theory of disordered materials [40] likewise deals with systems, e.g., glasses, of great conceptual similarity. Condensed matter theory can also serve as a reminder that the primary role of theory is not quantitative description, but rather qualitative 
understanding; anybody suspecting that not much useful can come of such a role should have a close look at the triumphs of condensed matter theory.

The beauty of theoretical protein science stems from its rapidly increasing treasure of new structures and functions; one could hardly imagine a science with greater riches in new discoveries and, hence, new challenges and with more relevance to the existence and well-being of humans.

\section{Acknowledgements}

The authors express their great indebtedness to Dong Xu who, together with K.S., had started the research on temperature echoes in our group, who had provided us with needed simulation programs and who had introduced H.L. to molecular dynamics of temperature echoes. The authors like to thank also Jim Phillips for help with the preparation and the revision of the manuscript. This work has been supported by the National Institutes of Health grant P41RRO5969.

\section{References}

[1] Neil W. Ashcroft and N. David Mermin. Solid State Physics. W. B. Saunders, Philadelphia, 1976.

[2] Rita G. Lerner and George L. Trigg. Encyclopedia of Physics. VCH Publisher, Inc., New York, 2 edition, 1990.

[3] T. Noguti and N. Gō. Collective variable description of small-amplitude conformational fluctuations in a globular protein. Nature, 296:776, 1982.

[4] B. R. Brooks and M. Karplus. Harmonic dynamics of proteins:normal modes and fluctuations in bovine pancreatic trypsin inhibitor. Proc. Natl. Acad. Sci. USA, 80:6571-6575, 1983.

[5] M. Levitt, C. Sander, and P. S. Stern. Protein normal-mode dynamics: Trypsin inhibitor, crambin, ribonuclease and lysozyme. J. Mol. Biol., 181:423-447, 1985.

[6] N Gō, T. Noguti, and T. Nishikawa. Dynamics of a small globular protein, in terms of low-frequency vibrational modes. Proc. Natl. Acad. Sci. USA, 80:3696, 1983.

[7] R. M. Levy, A. R. Srinivasan, W. K. Olson, and J. A. McCammon. Quasi-harmonic method for studying very low frequency modes in proteins. Biopolymers, 23:1099, 1984.

[8] R. M. Levy, O. de la Luz Rojas, and R. A. Friesner. Quasi-harmonic method for calculating vibrational spectra from classical simulations on multidimensional anharmonic potential surfaces. J. Phys. Chem., 88:4233, 1984.

[9] E.R. Henry, W.R. Eaton, and R.M. Hochstrasser. Molecular dynamics simulations of cooling in laser-excited heme proteins. Proc. Natl. Acad. Sci. USA, 83:8982-8986, 1986.

[10] J.F. Gibrat and N. Gō. Normal mode analysis of human lysozyme: Study of the relative motion of the two domains and characterization of harmonic motion. Proteins: Structure, Function, and Genetics, 8:258-279, 1990.

[11] B. R. Brooks and M. Karplus. Normal modes for specific motions of macromolecules: Application to the hinge-bending mode of lysozyme. Proc. Natl. Acad. Sci. USA, 82:4995, 1985. 
[12] William Humphrey, Ilya Logunov, Klaus Schulten, and Mordechai Sheves. Molecular dynamics study of bacteriorhodopsin and artificial pigments. Biochemistry, 33:3668-3678, 1994.

[13] G. S. Grest, S. R. Nagel, and A. Rahman. Quench echoes in molecular dynamics - a new phonon spectroscopy. Solid State Communications, 36:875-879, 1980.

[14] G. S. Grest, S. R. Nagel, A. Rahman, and T. W. Witten, Jr. Density of states and the velocity autocorrelation function derived from quench studies. J. Chem. Phys., 74(6):3532-3534, 1981.

[15] S. R. Nagel, A. Rahman, and G. S. Grest. Normal-mode analysis by quench-echo techniques: Localization in a amorphous solid. Phys. Rev. Lett., 47(23):1665-1668, 1981.

[16] S. R. Nagel, G. S. Grest, and A. Rahman. Quench echoes. Physics Today, October:24-32, 101983.

[17] O. M. Becker and M. Karplus. Temperature echoes in molecular dynamics simulations of proteins. Phys. Rev. Lett., 70(22):3514-3517, 1993.

[18] Dong $\mathrm{Xu}$, Klaus Schulten, Oren M. Becker, and Martin Karplus. Temperature quench echoes in proteins. J. Chem. Phys., 103:3112-3123, 1995.

[19] Dong Xu and Klaus Schulten. Velocity reassignment echoes in proteins. J. Chem. Phys., 103:3124-3139, 1995.

[20] Axel T. Brünger. X-PLOR. The Howard Hughes Medical Institute and Department of Molecular Biophysics and Biochemistry, Yale University, New Haven, CT, May 1988.

[21] Bernard R. Brooks, Robert E. Bruccoleri, Barry D. Olafson, David J. States, S. Swaminathan, and Martin Karplus. CHARMm: a program for macromolecular energy, minimization, and dynamics calculations. J. Comp. Chem., 4(2):187-217, 1983.

[22] S. Lifson and P. S. Stern. Born-oppenheimer energy surfaces of similar molecules - interrelations between bond lengths, bond angles, and frequencies of normal vibrations in alkanes. J. Chem. Phys., 77:4542-4550, 1982.

[23] Lord Rayleigh. Scientific Papers, Vol. I, p491 and Vol. IV, p370. Cambridge University Press, Cambridge, England, 1899-1920.

[24] Lord Rayleigh. The Theory of Sound, Vol. I, 2nd ed. MacMillan and Company Ltd., London, 1894.

[25] Andreas Windemuth and Klaus Schulten. Stochastic dynamics simulation for macromolecules. Beckman Institute Technical Report TB-91-19, University of Illinois, 1991.

[26] C. W. Gardiner. Handbook of Stochastic Methods. Springer, New York, 1983.

[27] H. Risken. The Fokker-Planck Equation: Methods of Solution and Applications, 2nd Ed. Springer, New York, 1989.

[28] N. G. van Kampen. Stochastic Processes in Physics and Chemistry. North-Holland, Amsterdam, New York, 1992.

[29] H. Frauenfelder, F. Parak, and R.D. Young. Conformational substates in proteins. Ann. Rev. Biophys. Biophys. Chem., 17:451-79, 1988.

[30] B. Cartling. From short-time molecular dynamics to long-time stochastic dynamics of proteins. J. Chem. Phys., 91:427-438, 1989.

[31] Bo Cartling. Stochastic model of intermode couplings in protein dynamics. J. Chem. Phys., 94:6203-6210, 1991.

[32] S. Chandrasekhar. Stochastic problems in physics and astronomy. Rev. Mod. Phys., 15:1-89, 1943. 
[33] M. C. Wang and G. E. Uhlenbeck. On the theory of the brownian motion II. Rev. Mod. Phys., 17(2):323-342, 1945.

[34] Ch. Jungnickel and R. Mc Cormmach. Intellectual Mastery of Nature - Theoretical Physics from Ohm to Einstein, Vol. 1: The Torch of Mathematics 1800-1870. Chicago University Press, Chicago, 1986.

[35] Ch. Jungnickel and R. Mc Cormmach. Intellectual Mastery of Nature - Theoretical Physics from Ohm to Einstein, Vol. 2: The Now Mighty Theoretical Physics 18701925. Chicago University Press, Chicago, 1986.

[36] R. Car and M. Parrinello. Structual, dynamics and electronic properties of amorphous silicon, an ab initio molecular-dynamics study. Phys. Rev. Lett., 60:204-207, 1988.

[37] Helmut Grubmüller, Helmut Heller, Andreas Windemuth, and Klaus Schulten. Generalized Verlet algorithm for efficient molecular dynamics simulations with longrange interactions. Molecular Simulation, 6:121-142, 1991.

[38] M. P. Allen and D. J. Tildesley. Computer Simulation of Liquids. Oxford University Press, New York, 1987.

[39] J. P. Boon and S. Yip. Molecular Hydrodynamics. Dover Publication, Inc., New York, 1980.

[40] N. E. Cusack. The Physics of Structurally Disordered Matter. Adam Hilger, Bristol, 1987. 